Review

\title{
Spin Exchanges between Transition Metal Ions Governed by the Ligand p-Orbitals in Their Magnetic Orbitals
}

\author{
Myung-Hwan Whangbo ${ }^{1,2, * \mathbb{D}}$, Hyun-Joo Koo ${ }^{1}$ and Reinhard K. Kremer ${ }^{3} \mathbb{D}$ \\ 1 Department of Chemistry and Research Institute for Basic Sciences, Kyung Hee University, \\ Seoul 02447, Korea; hjkoo@khu.ac.kr \\ 2 Department of Chemistry, North Carolina State University, Raleigh, NC 27695-8204, USA \\ 3 Max Planck Institute for Solid State Research, Heisenbergstrasse 1, D-70569 Stuttgart, Germany; \\ rekre@fkf.mpg.de \\ * Correspondence: whangbo@ncsu.edu; Tel.: +0f1-919-515-3464
}

check for updates

Citation: Whangbo, M.-H.; Koo, H.-J.; Kremer, R.K. Spin Exchanges between Transition Metal Ions Governed by the Ligand p-Orbitals in Their Magnetic Orbitals. Molecules 2021, 26, 531. https://doi.org/ $10.3390 /$ molecules 26030531

Academic Editors:

Alessandro Stroppa and Igor Djerdj

Received: 19 December 2020

Accepted: 15 January 2021

Published: 20 January 2021

Publisher's Note: MDPI stays neutral with regard to jurisdictional claims in published maps and institutional affiliations.

Copyright: (c) 2021 by the authors. Licensee MDPI, Basel, Switzerland. This article is an open access article distributed under the terms and conditions of the Creative Commons Attribution (CC BY) license (https:// creativecommons.org/licenses/by/ $4.0 /)$.

\begin{abstract}
In this review on spin exchanges, written to provide guidelines useful for finding the spin lattice relevant for any given magnetic solid, we discuss how the values of spin exchanges in transition metal magnetic compounds are quantitatively determined from electronic structure calculations, which electronic factors control whether a spin exchange is antiferromagnetic or ferromagnetic, and how these factors are related to the geometrical parameters of the spin exchange path. In an extended solid containing transition metal magnetic ions, each metal ion $\mathrm{M}$ is surrounded with main-group ligands $\mathrm{L}$ to form an $M L_{n}$ polyhedron (typically, $n=3-6$ ), and the unpaired spins of $M$ are represented by the singly-occupied d-states (i.e., the magnetic orbitals) of $\mathrm{ML}_{n}$. Each magnetic orbital has the metal d-orbital combined out-of-phase with the ligand p-orbitals; therefore, the spin exchanges between adjacent metal ions $\mathrm{M}$ lead not only to the M-L-M-type exchanges, but also to the M-L ... L-M-type exchanges in which the two metal ions do not share a common ligand. The latter can be further modified by $\mathrm{d}^{0}$ cations $\mathrm{A}$ such as $\mathrm{V}^{5+}$ and $\mathrm{W}^{6+}$ to bridge the $\mathrm{L}$... L contact generating M-L ... A ... L-M-type exchanges. We describe several qualitative rules for predicting whether the M-L . . L L-M and M-L . . A . . L-M-type exchanges are antiferromagnetic or ferromagnetic by analyzing how the ligand p-orbitals in their magnetic orbitals (the ligand p-orbital tails, for short) are arranged in the exchange paths. Finally, we illustrate how these rules work by analyzing the crystal structures and magnetic properties of four cuprates of current interest: $\alpha-\mathrm{CuV}_{2} \mathrm{O}_{6}, \mathrm{LiCuVO}_{4}$, $(\mathrm{CuCl}) \mathrm{LaNb}_{2} \mathrm{O}_{7}$, and $\mathrm{Cu}_{3}\left(\mathrm{CO}_{3}\right)_{2}(\mathrm{OH})_{2}$.
\end{abstract}

Keywords: Keywords: spin exchange; magnetic orbitals; ligand p-orbital tails; $\mathrm{M}-\mathrm{L}-\mathrm{M}$ exchange; $\mathrm{M}-\mathrm{L} \ldots$. L-M exchange; $\alpha-\mathrm{CuV}_{2} \mathrm{O}_{6} ; \mathrm{LiCuVO}_{4} ;(\mathrm{CuCl}) \mathrm{LaNb}_{2} \mathrm{O}_{7} ; \mathrm{Cu}_{3}\left(\mathrm{CO}_{3}\right)_{2}(\mathrm{OH})_{2}$

\section{Introduction}

An extended solid consisting of transition metal magnetic ions has closely packed energy states (Figure 1a,b) so that, at a given non-zero temperature, the ground state as well as a vast number of the excited states can be thermally occupied. The thermodynamic properties such as the magnetic susceptibility and the specific heat of a magnetic system represents the weighted average of the properties associated with all thermally occupied states, with their Boltzmann factors as the weights. Such a quantity is difficult to calculate if all states were to be determined by first principle electronic structure calculations.

To generate the states of a given magnetic system and subsequently calculate the thermally-averaged physical property, a model Hamiltonian (also called a toy Hamiltonian) is invariably employed [1-3]. A typical model Hamiltonian used for this purpose is the Heisenberg-type spin Hamiltonian, $\mathrm{H}_{\text {spin }}$, expressed as:

$$
\mathrm{H}_{\text {spin }}=\sum_{\mathrm{i}>\mathrm{j}} \mathrm{J}_{\mathrm{ij}} \hat{\mathrm{S}}_{\mathrm{i}} \cdot \hat{\mathrm{S}}_{\mathrm{j}}
$$


where the energy spectrum of a magnetic system as the sum of the pairwise spin exchange interactions $J_{i j} \hat{S}_{i} \cdot \hat{S}_{j}$ is approximated. The spin operators $\hat{S}_{i}$ and $\hat{S}_{j}$ (at the spin sites $i$ and $j$, respectively) can be treated as the spin vectors $\vec{S}_{i}$ and $\vec{S}_{j}$, respectively, unless they operate on spin states. If all magnetic ions of a given system are identical with spin $S$, each term $\vec{S}_{\mathrm{i}} \cdot \vec{S}_{\mathrm{j}}$ can be written as $\vec{S}_{\mathrm{i}} \cdot \vec{S}_{\mathrm{j}}=\mathrm{S}^{2} \cos \theta_{\mathrm{ij}}$ where $\theta_{\mathrm{ij}}$ is the angle between the two spin vectors. In such a case, Equation (1) is rewritten as:

$$
\mathrm{H}_{\text {spin }}=\sum_{\mathrm{i}>\mathrm{j}} \mathrm{J}_{\mathrm{ij}} \mathrm{S}^{2} \cos \theta_{\mathrm{ij}}
$$

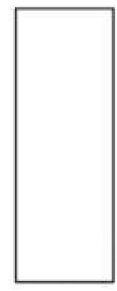

(a)

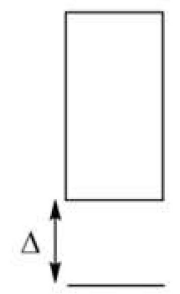

(b) (c)

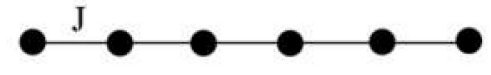

(d)

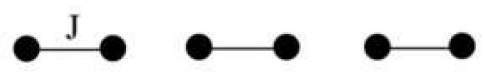

(e)

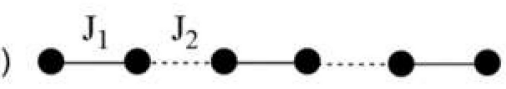

Figure 1. $(\mathbf{a}, \mathbf{b})$ Allowed energy states of a magnetic solid. Between the lowest-lying excited state and the ground state, there is no energy gap in (a), but a non-zero energy gap in (b). (c-e) Examples of simple spin lattices: a uniform chain in (c); isolated spin dimers in (d); and an alternating chain in (e). Here, all nearest-neighbor spins are antiferromagnetically coupled.

In a collinearly ordered spin arrangement of a magnetic solid, every two spin arrangements are either ferromagnetic $\left(\mathrm{FM}\right.$, i.e., parallel $\left.\left(\theta_{\mathrm{ij}}=0^{\circ}\right)\right)$ or antiferromagnetic (AFM, i.e., antiparallel $\left(\theta_{\mathrm{ij}}=180^{\circ}\right)$ ). With the definition of a spin Hamiltonian as in Equation (1), AFM and FM spin exchanges are represented by positive and negative $\mathrm{J}_{\mathrm{ij}}$ values, respectively. For any collinearly ordered spin arrangement, the total energy is readily written as a function of the various spin exchanges $\mathrm{J}_{\mathrm{ij}}$.

The energy spectrum allowed for a magnetic system, and hence its magnetic properties, depend on its spin lattice. The latter refers to the repeat pattern of predominant spin exchange paths, i.e., those with large $\left|\mathrm{J}_{\mathrm{ij}}\right|$ values. For example, between the ground and the excited states, a uniform half-integer spin AFM chain (Figure 1c) has no energy gap (Figure 1a), whereas an isolated AFM dimer (Figure 1d) and an alternating AFM chain (Figure 1e) have a non-zero energy gap (Figure $1 \mathrm{~b}$ ). The spin lattice of a given magnetic solid is determined by its electronic structure, which makes it interesting how to identify the spin lattice of a magnetic solid on the basis of its atomic and electronic structures. Strong AFM exchanges between magnetic ions are often termed magnetic bonds, in contrast to chemical bonds determined by strong chemical bonding. Thus, Figure 1c represents a uniform AFM chain, and Figure 1d represents isolated AFM dimers. The magnetic bonds do not necessarily follow the geometrical pattern of the magnetic ion arrangement dictated by chemical bonding.

In a magnetic solid, transition metal ions $M$ often share a common ligand $L$ to form M-L-M bridges (Figure 2a). The spin exchange between the magnetic ions in an M-L-M bridge has been termed "superexchange" [4-7]. Whether the spin arrangement between two metal ions becomes FM or AFM, as described by the Goodenough-Kanamori rules formulated in the late 1950s, depends on the geometry of the M-L-M bridge [5-9]. Ever since, the Goodenough-Kanamori rules have greatly influenced the thinking of inorganic and solid-state chemists dealing with magnetic systems. Since the early 2000s, it has become increasingly clear that the magnetic properties of certain compounds cannot be conclusively explained unless also one takes into consideration the spin exchanges of the M-L . . L-M (Figure 2b) or the M-L ... A ... L-M (Figure 2c) types, termed super-superexchanges [1-3], 
in which the metal ions do not share a common ligand. In the late 1950s, it was impossible to imagine that spin exchanges could take place in such paths in which the M ... M distances were very long, because the prevailing concept of chemical bonding at that time, mainly based on the valence bond picture [10], suggested that an unpaired electron of a magnetic ion is accommodated in a pure d-orbital of $\mathrm{M}$. In an extended solid, each transition metal ion $M$ is typically surrounded with main-group ligand atoms $L$ to form an $M L_{n}$ polyhedron $(n=3-6)$, and each unpaired electron of $M L_{n}$ resides in a singly-occupied $\mathrm{d}$-state (referred to as a magnetic orbital) of $\mathrm{ML}_{n}$, in which the d-orbital of $\mathrm{M}$ is combined out-of-phase with the p-orbitals of L. In this molecular orbital picture, the unpaired spin density is already delocalized from the d-orbital of $M$ (the "magnetic orbital head") to the p-orbitals of L (the "magnetic orbital tails") [1-3]. Thus, it is quite natural to think that the spin exchange between the metal ions in an M-L ... L-M path takes place through the overlap between the ligand p-orbital tails present in the L ... L contact. This interaction between the p-orbital tails can be modified by the empty $\mathrm{d}_{\pi}$ orbitals of the $\mathrm{d}^{0}$ cation $\mathrm{A}$ (e.g., $\mathrm{V}^{5+}$ and $\mathrm{W}^{6+}$ ) in an M-L ... A ... L-M exchange.

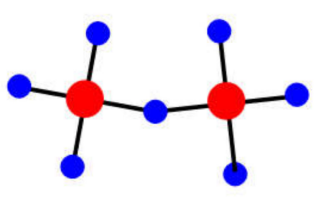

(a) $M-L-M$

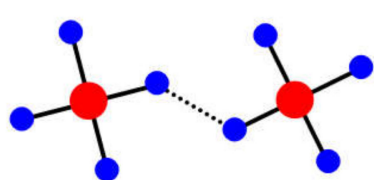

(b) $M-L \ldots L-M$

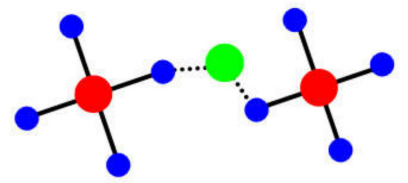

(c) $M-L \ldots A \ldots L-M$

Figure 2. Three types of spin exchange paths associated with two magnetic ions: (a) M-L-M, (b) M-L ... L-M, and (c) M-L ... A ... L-M, where A represents a $\mathrm{d}^{0}$ cation such as $\mathrm{V}^{5+}$ or $\mathrm{W}^{6+}$. The $\mathrm{M}, \mathrm{L}$, and $\mathrm{A}$ are represented by red, blue, and green circles, respectively.

Given that spin exchanges between magnetic ions are determined by the p-orbital tails of their magnetic orbitals, it is not surprising that spin exchanges of the $\mathrm{M}-\mathrm{L} \ldots$ $\mathrm{L}-\mathrm{M}$ or $\mathrm{M}-\mathrm{L}$.. . A . . . L-M type can be stronger than those of the $\mathrm{M}-\mathrm{L}-\mathrm{M}$ type, and that spin exchanges are generally strong only along a certain direction of the crystal structure. Although this aspect has been repeatedly pointed out in review articles over the years [1-3], it is still not infrequent to observe that experimental results are incorrectly interpreted simply because spin lattices have been deduced considering only the $\mathrm{M}-\mathrm{L}-\mathrm{M}$ type spin exchanges. Such an unfortunate mishap is akin to providing solutions in search of a problem. In this review, written as a tribute to John B. Goodenough for his long and illustrious scientific career culminating with the Nobel Prize in 2019, we review what electronic factors govern the nature of the $\mathrm{M}-\mathrm{L}-\mathrm{M}, \mathrm{M}-\mathrm{L} \ldots \mathrm{L}-\mathrm{M}$ and $\mathrm{M}-\mathrm{L}$. . . A . . L $-\mathrm{M}$ type spin exchanges, with an ultimate goal to provide several qualitative rules useful for finding the spin lattice relevant for any given magnetic system.

Our work is organized as follows: Section 2 examines how to quantitatively determine the values of spin exchanges by carrying out the energy-mapping analysis based on electronic structure calculations. Section 3 explores the electronic factors controlling whether a spin exchange is AFM or FM. In Section 4, we derive several qualitative rules that enable one to predict whether the M-L . . L-M and M-L . . A . . L-M type spin exchanges are AFM or FM by analyzing how their ligand p-orbitals are arranged in the exchange paths. To illustrate how the resulting structure-property relationships operate, in Section 5 we examine the crystal structures and magnetic properties of $\alpha-\mathrm{CuV}_{2} \mathrm{O}_{6}, \mathrm{LiCuVO}{ }_{4},(\mathrm{CuCl}) \mathrm{LaNb}_{2} \mathrm{O}_{7}$ and $\mathrm{Cu}_{3}\left(\mathrm{CO}_{3}\right)_{2}(\mathrm{OH})_{2}$. Our concluding remarks are presented in Section 6.

\section{Energy Mapping Analysis for Quantitative Evaluation of Spin Exchanges}

This section probes how to define the relevant spin Hamiltonian for a given magnetic system on a quantitative level. This requires the determination of the spin exchanges to include in the spin Hamiltonian. For various collinearly ordered spin states of a given 
magnetic system, one finds the expressions for their relative energies in terms of the spin exchange parameters $\mathrm{J}_{\mathrm{ij}}$ to evaluate, performs $\mathrm{DFT}+\mathrm{U}$ [11] or DFT+hybrid [12] electronic structure calculations for the ordered spin states to determine the numerical values for their relative energies, and finally maps the two sets of relative energies to find the numerical values of the exchanges.

\subsection{Using Eigenstates}

To gain insight into the meaning and the nature of a spin exchange, we examine a spin dimer made up of two $S=1 / 2$ ions (Figure 3a). The spin Hamiltonian describing the energies of this dimer is given by:

$$
\mathrm{H}_{\mathrm{spin}}=\mathrm{J} \hat{\mathrm{S}}_{\mathrm{i}} \cdot \hat{\mathrm{S}}_{\mathrm{j}} \text {, }
$$

(a)

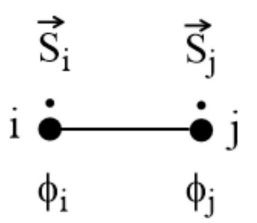

(b)

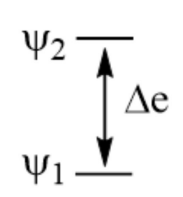

(c)

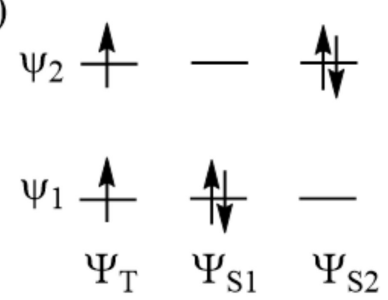

Figure 3. (a) Spin dimer made up of two $S=1 / 2$ ions at sites $i$ and $j$. Each site has one unpaired spin. The magnetic orbitals at the sites $i$ and $j$ are represented by $\phi_{i}$ and $\phi_{j}$, respectively. (b) The bonding and antibonding states, $\Psi_{1}$ and $\Psi_{2}$, resulting from the interactions between $\phi_{\mathrm{i}}$ and $\phi_{\mathrm{j}}$, are split in energy by $\Delta \mathrm{e}$. (c) The singlet and triplet electron configurations resulting from $\Psi_{1}$ and $\Psi_{2}$.

The spin states allowed for this dimer are the singlet and triplet states, $\Psi_{\mathrm{S}}$ and $\Psi_{\mathrm{T}}$, respectively.

$$
\begin{gathered}
\Psi_{\mathrm{T}}=|\uparrow \uparrow\rangle,|\downarrow \downarrow\rangle,(|\uparrow \downarrow\rangle+|\downarrow \uparrow\rangle) / \sqrt{2} \\
\Psi_{\mathrm{S}}=(|\uparrow \downarrow\rangle-|\downarrow \uparrow\rangle) / \sqrt{2}
\end{gathered}
$$

It can be readily shown that these states are the eigenstates of the spin Hamiltonian by rewriting Equation (3) as:

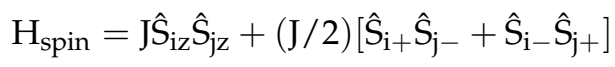

where $\hat{S}_{m z}(m=i, j)$ is the z-component of $\hat{S}_{m}$, while $\hat{S}_{m+}$ and $\hat{S}_{m-}$ are the raising and lowering operators associated with $\hat{S}_{\mathrm{m}}$, respectively. Then, it is found that:

$$
\begin{gathered}
\mathrm{H}_{\text {spin }} \Psi_{\mathrm{T}}=(\mathrm{J} / 4) \Psi_{\mathrm{T}} \\
\mathrm{H}_{\text {spin }} \Psi_{\mathrm{S}}=(-3 \mathrm{~J} / 4) \Psi_{\mathrm{S}}
\end{gathered}
$$

Thus:

$$
\Delta \mathrm{E}_{\text {eigen }}=\mathrm{E}_{\mathrm{T}}-\mathrm{E}_{\mathrm{S}}=\left\langle\Psi_{\mathrm{T}}\left|\mathrm{H}_{\text {spin }}\right| \Psi_{\mathrm{T}}\right\rangle-\left\langle\Psi_{\mathrm{S}}\left|\mathrm{H}_{\text {spin }}\right| \Psi_{\mathrm{S}}\right\rangle=\mathrm{J}
$$

Therefore, the spin exchange $\mathrm{J}$ is related to the energy difference between the singlet and triplet states of the spin dimer, as illustrated in Figure $4 a$. 


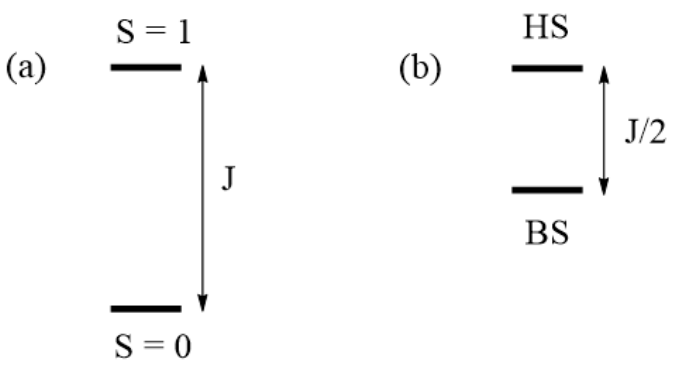

Figure 4. Relationships of the spin exchange $\mathrm{J}$ to the energy difference between two spin states of a spin dimer made up of two $S=1 / 2$ ions in terms of (a) the eigenstates and (b) the broken-symmetry states. The legends HS and BS in (b) refer to the high-symmetry and broken-symmetry states, respectively.

\subsection{Using Broken-Symmetry States}

In general, it is not an easy task to find the eigenstates of a general spin Hamiltonian (e.g., Equation (1)), which makes it difficult to relate the spin exchanges to the energy differences between the eigenstates of a magnetic system. However, the energies for the brokensymmetry (BS) states (i.e., the non-eigenstates) of a spin Hamiltonian are easy to evaluate. For the magnetic dimer in Section 2.1, the high-spin (HS) state, $\Psi_{\mathrm{HS}}=|\uparrow \uparrow\rangle$ or $|\downarrow \downarrow\rangle$, which has an FM spin arrangement, is an eigenstate of the spin Hamiltonian (Equation (3)). However, the low-spin (LS) state can be expressed as:

$$
\Psi_{\mathrm{LS}}=|\uparrow \downarrow\rangle \text { or }|\downarrow \uparrow\rangle
$$

This state has an AFM spin arrangement and is the BS state of the spin Hamiltonian. Using Equation (5), it is found that:

$$
\begin{aligned}
& \mathrm{H}_{\text {spin }}|\uparrow \downarrow\rangle=(-\mathrm{J} / 4)|\uparrow \downarrow\rangle+(\mathrm{J} / 2)|\downarrow \uparrow\rangle \\
& \mathrm{H}_{\text {spin }}|\downarrow \uparrow\rangle=(-\mathrm{J} / 4)|\downarrow \uparrow\rangle+(\mathrm{J} / 2)|\uparrow \downarrow\rangle
\end{aligned}
$$

Therefore:

$$
\left\langle\uparrow \downarrow\left|\mathrm{H}_{\text {spin }}\right| \uparrow \downarrow\right\rangle=\left\langle\downarrow \uparrow\left|\mathrm{H}_{\text {spin }}\right| \downarrow \uparrow\right\rangle=-\mathrm{J} / 4
$$

From Equations (6) and (9), we obtain:

$$
\Delta \mathrm{E}_{\mathrm{BS}}=\left\langle\Psi_{\mathrm{HS}}\left|\mathrm{H}_{\text {spin }}\right| \Psi_{\mathrm{HS}}\right\rangle-\left\langle\Psi_{\mathrm{BS}}\left|\mathrm{H}_{\text {spin }}\right| \Psi_{\mathrm{BS}}\right\rangle=\mathrm{J} / 2
$$

The spin exchange $\mathrm{J}$ is related to the energy difference between the states including the BS states (Figure $4 b$ ).

\subsection{Energy Mapping}

For a general spin lattice, the energy difference between any two states (involving BS and HS states) can be readily determined by using the spin Hamiltonian of Equation (3), which is expressed as a function of unknown constants $\mathrm{J}_{\mathrm{ij}}$. To evaluate the spin exchanges $\mathrm{J}_{\mathrm{ij}}$ of a general spin Hamiltonian, it is necessary to numerically determine the relative energies of various ordered spin states. This is done by performing DFT+U [11] or DFT+hybrid [12] electronic structure calculations for the collinearly ordered spin states of a magnetic system on the basis of the electronic Hamiltonian, $\mathrm{H}_{\text {elec }}$. These types of calculations ensure that the electronic structures calculated for various ordered spin states have a bandgap as expected for a magnetic insulator. Suppose that $\mathrm{N}$ different spin exchange paths $\mathrm{J}_{\mathrm{ij}}$ are considered to describe a given magnetic solid. If one considers $\mathrm{N}+1$ ordered spin states, for example, one can determine $\mathrm{N}$ different relative energies $\Delta \mathrm{E}_{\text {spin }}(\mathrm{i})(\mathrm{i}=1,2, \cdots, \mathrm{N})$ expressed in terms of $\mathrm{N}$ different spin exchanges $\mathrm{J}_{\mathrm{ij}}$ (in principle, one could use more spin states, but at least $\mathrm{N}+1$ are necessary; using more would produce error bars and increase the precision of the analysis). By carrying out electronic structure calculations for the $\mathrm{N}+1$ ordered spin states of the magnetic system, one obtains the numerical values for the $\mathrm{N}$ different relative energies $\Delta \mathrm{E}_{\text {elec }}(\mathrm{i})(\mathrm{i}=1,2, \cdots, N)$. Then, by equating the $\Delta \mathrm{E}_{\text {spin }}(\mathrm{i})(\mathrm{i}=1,2, \cdots, N)$ values 
to the corresponding $\Delta \mathrm{E}_{\mathrm{elec}}(\mathrm{i})(\mathrm{i}=1,2, \cdots, \mathrm{N})$ values, the $\mathrm{N}$ different spin exchanges $\mathrm{J}_{\mathrm{ij}}$ are obtained.

$$
\Delta \mathrm{E}_{\text {spin }}(\mathrm{i})(\mathrm{i}=1,2, \cdots, \mathrm{N}) \leftrightarrow \Delta \mathrm{E}_{\text {elec }}(\mathrm{i})(\mathrm{i}=1,2, \cdots, \mathrm{N})
$$

The spin exchanges discussed so far are known as Heisenberg exchanges. There are other variants of interactions between spins which, though weaker than Heisenberg exchanges in strength, are needed to explain certain magnetic properties not covered by the symmetrical Heisenberg exchanges. They include Dzyaloshinskii-Moriya exchanges (or antisymmetric exchanges) and asymmetric exchanges [2,3]. The energy-mapping analysis based on collinearly ordered spin states allows one to determine the Heisenberg spin exchanges only. To evaluate the Dzyaloshinskii-Moriya and asymmetric spin exchanges, the energy-mapping analysis employs the four-state method [2,13], in which non-collinearly ordered broken-symmetry states are used. A further generalization of this energy-mapping method was developed to enable the evaluation of other energy terms that one might include in a model spin Hamiltonian [14].

\section{Qualitative Features of Spin Exchange}

A spin Hamiltonian appropriate for a given magnetic system is one that consists of the predominant spin exchange paths. Such a spin Hamiltonian can be determined by evaluating the values of various possible spin exchanges for the magnetic system by performing the energy-mapping analysis as described in Section 2. If the spin lattice is chosen without quantitatively evaluating its spin exchanges, one might inadvertently choose a spin lattice irrelevant for the interpretation of the experimental data. When simulating the thermodynamic properties using a chosen set of spin exchanges, the values of the spin exchanges are optimized until they provide the best possible simulation even if the chosen spin lattice is incorrect from the viewpoint of electronic structure. Thus, in principle, more than one spin lattice might provide an equally good simulation. In interpreting the experimental results of a magnetic system with a correct spin lattice, it is crucial to know what electronic and structural factors control the signs and the magnitudes of spin exchanges.

\subsection{Parameters Affecting Spin Exchanges}

To examine what energy parameters govern the sign and magnitude of a spin exchange, we revisit the spin exchange of the spin dimer (Figure 3a) by explicitly considering the electronic structures of its singlet and triplet states. For simplicity, we represent each spin site with one magnetic orbital. As will be discussed in Section 5, the nature of the magnetic orbital plays a crucial role in determining the sign and magnitude of a spin exchange. We label the magnetic orbitals located at the spin sites $i$ and $j$ as $\phi_{i}$ and $\phi_{j}$, respectively (Figure 3a). These orbitals overlap weakly, and hence interact weakly, to form the in-phase and out-of-phase states, $\Psi_{1}$ and $\Psi_{2}$, respectively, with energy split $\Delta$ e between the two (Figure 3b). The overlap integral $\left\langle\phi_{\mathrm{i}} \mid \phi_{\mathrm{j}}\right\rangle$ between $\phi_{\mathrm{i}}$ and $\phi_{\mathrm{j}}$ is small for magnetic systems, so $\Psi_{1}$ and $\Psi_{2}$ are well approximated by:

$$
\Psi_{1} \approx \frac{\left(\phi_{\mathrm{i}}+\phi_{\mathrm{j}}\right)}{\sqrt{2}}, \Psi_{2} \approx \frac{\left(\phi_{\mathrm{i}}-\phi_{\mathrm{j}}\right)}{\sqrt{2}}
$$

For simplicity, it is assumed here that the in-phase combination (i.e., the bonding combination) is described by the plus combination, which amounts to the assumption that the overlap integral $\left\langle\phi_{\mathrm{i}} \mid \phi_{\mathrm{j}}\right\rangle$ is positive. The energy split $\Delta \mathrm{e}$ is approximately proportional to the overlap integral:

$$
\Delta \mathrm{e} \propto\left\langle\phi_{\mathrm{i}} \mid \phi_{\mathrm{j}}\right\rangle
$$


In understanding the qualitative features describing the electronic energy difference between the singlet and triplet states, $\Delta \mathrm{E}_{\text {elec }}$, and hence the spin exchange $\mathrm{J}$, it is necessary to consider two other quantities. One is the on-site repulsion $\mathrm{U}_{\mathrm{ii}}$ :

$$
\mathrm{U}_{\mathrm{ii}}=\left\langle\phi_{\mathrm{i}}(1) \phi_{\mathrm{i}}(2)\left|1 / \mathrm{r}_{12}\right| \phi_{\mathrm{i}}(1) \phi_{\mathrm{i}}(2)\right\rangle(\mathrm{i}=1,2)
$$

where the product $\phi_{\mathrm{i}}(\mathrm{m}) \phi_{\mathrm{i}}(\mathrm{m})$ is the electron density $\rho_{\mathrm{ii}}(\mathrm{m})$ associated with the orbital $\phi_{\mathrm{i}}(\mathrm{m})$ occupied by electron $\mathrm{m}(=1,2)$. Thus, $\mathrm{U}_{\mathrm{ii}}$ is the self-repulsion when the orbital $\phi_{\mathrm{i}}$ is occupied by two electrons. If the spin sites $i$ and $j$ are identical in nature, the on-site repulsion $U_{\mathrm{jj}}$ at the site $\mathrm{j}$ is the same as $\mathrm{U}_{\mathrm{ii}}$, therefore it is convenient to use the symbol $\mathrm{U}$ to represent both $\mathrm{U}_{\mathrm{ii}}$ and $\mathrm{U}_{\mathrm{jj}}$ (i.e., $\mathrm{U}=\mathrm{U}_{\mathrm{ii}}=\mathrm{U}_{\mathrm{jj}}$ ). The other quantity of interest is the exchange repulsion $\mathrm{K}_{\mathrm{ij}}$ between $\phi_{\mathrm{i}}$ and $\phi_{\mathrm{j}}$ :

$$
K_{\mathrm{ij}}=\left\langle\phi_{\mathrm{i}}(1) \phi_{\mathrm{j}}(2)\left|1 / \mathrm{r}_{12}\right| \phi_{\mathrm{j}}(1) \phi_{\mathrm{i}}(2)\right\rangle
$$

This is the self-repulsion arising from the overlap electron density:

$$
\rho_{\mathrm{ij}}(\mathrm{m})=\phi_{\mathrm{i}}(\mathrm{m}) \phi_{\mathrm{j}}(\mathrm{m})(\mathrm{m}=1,2)
$$

To illustrate the difference between the overlap integral and overlap electron density, we consider the $p_{x}$ and $p_{y}$ atomic orbitals located at a same atomic site (Figure 5a,b). The product $p_{x} p_{y}$ represents the overlap electron density $\rho_{x y}$, which consists of four overlapping regions (Figure $5 \mathrm{c}$ ); two regions of positive electron density (colored in pink) and two regions of negative electron density (colored in cyan). The overlap integral $\left\langle p_{x} \mid p_{y}\right\rangle$ is the sum of these four overlap electron densities, which adds up to zero.

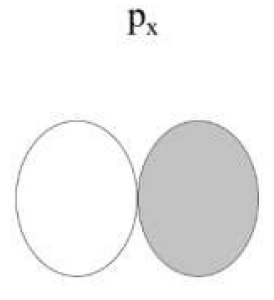

(a)

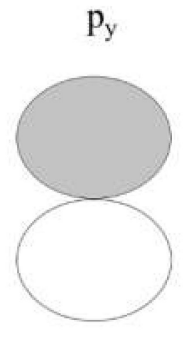

(b)

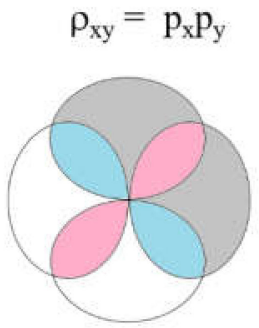

(c)

Figure 5. The overlap density resulting from two p-orbital at a given atomic site: (a) a $\mathrm{p}_{\mathrm{x}}$ orbital; (b) a $p_{y}$ orbital; and (c) the overlap density between the two orbitals, $\rho_{x y}=p_{x} p_{y}$. The pink and cyan regions have positive and negative values, respectively.

The exchange repulsion between $\mathrm{p}_{\mathrm{x}}$ and $\mathrm{p}_{\mathrm{y}}$ is written as:

$$
\mathrm{K}_{\mathrm{xy}}=\left\langle\mathrm{p}_{\mathrm{x}}(1) \mathrm{p}_{\mathrm{x}}(2)\left|1 / \mathrm{r}_{12}\right| \mathrm{p}_{\mathrm{y}}(1) \mathrm{p}_{\mathrm{y}}(2)\right\rangle
$$

Equation (17) is given by the sum of the self-repulsion resulting from each overlapping region of the overlap electron density $\rho_{x y}$ (Figure $5 c$ ). Each overlapping region, be it positive or negative, leads to a positive repulsion; therefore, the exchange repulsion $\mathrm{K}_{\mathrm{xy}}$ is positive.

\subsection{Two Competing Components of Spin Exchange}

In Figure $3 c$, the configuration $\Psi_{\mathrm{T}}$ represents the triplet state of the dimer while the configurations $\Psi_{\mathrm{S} 1}$ and $\Psi_{\mathrm{S} 2}$ each represent a singlet state. For a magnetic system, for which $\Delta \mathrm{e}$ is very small, the singlet state is not well described by $\Psi_{\mathrm{S} 1}$ alone and is represented by a linear combination of $\Psi_{\mathrm{S} 1}$ and $\Psi_{\mathrm{S} 2}$, i.e., $\Psi_{\mathrm{S}}=\mathrm{c}_{1} \Psi_{\mathrm{S} 1}+\mathrm{c}_{2} \Psi_{\mathrm{S} 2}$, where the mixing coefficients $c_{1}$ and $c_{2}$ are determined by the interaction between $\Psi_{\mathrm{S} 1}$ and 
$\Psi_{\mathrm{S} 2}$, namely, $\left\langle\Psi_{\mathrm{S} 1}\left|\mathrm{H}_{\mathrm{elec}}\right| \Psi_{\mathrm{S} 2}\right\rangle=\mathrm{K}_{\mathrm{ij}}$, as well as by the energies of $\Psi_{\mathrm{S} 1}$ and $\Psi_{\mathrm{S} 2}$, that is, $\left\langle\Psi_{\mathrm{S} 1}\left|\mathrm{H}_{\text {elec }}\right| \Psi_{\mathrm{S} 1}\right\rangle=\mathrm{E}_{1}$ and $\left\langle\Psi_{\mathrm{S} 2}\left|\mathrm{H}_{\text {elec }}\right| \Psi_{\mathrm{S} 2}\right\rangle=\mathrm{E}_{2}$. After some lengthy manipulations under the condition, $K_{i j}>>\left|E_{1}-E_{2}\right|$, which is satisfied for magnetic systems, the energy difference between the triplet and singlet states, and hence the spin exchange $\mathrm{J}$, is expressed as [15]:

$$
\mathrm{J}=\mathrm{E}_{\mathrm{T}}-\mathrm{E}_{\mathrm{S}} \approx-2 \mathrm{~K}_{\mathrm{ij}}+\frac{(\Delta \mathrm{e})^{2}}{\mathrm{U}}
$$

Note that the spin exchange $\mathrm{J}$ consists of two components, $\mathrm{J}=\mathrm{J}_{\mathrm{F}}+\mathrm{J}_{\mathrm{AF}}$, where:

$$
\begin{aligned}
& \mathrm{J}_{\mathrm{F}}=-2 \mathrm{~K}_{\mathrm{ij}}<0 \\
& \mathrm{~J}_{\mathrm{AF}}=\frac{(\Delta)^{2}}{\mathrm{U}}>0
\end{aligned}
$$

The magnitude of the FM component $\left|\mathrm{J}_{\mathrm{F}}\right|$ increases as the exchange repulsion $\mathrm{K}_{\mathrm{ij}}$ increases, namely, as the overlap density $\left|\rho_{\mathrm{ij}}\right|=\left|\phi_{\mathrm{i}} \phi_{\mathrm{j}}\right|$ increases. The strength of the AFM component $\mathrm{J}_{\mathrm{AF}}$ increases with increasing the energy split $\Delta$ e, i.e., with increasing the overlap integral $\left\langle\phi_{\mathrm{i}} \mid \phi_{\mathrm{j}}\right\rangle$, while $\mathrm{J}_{\mathrm{AF}}$ decreases as the on-site repulsion $\mathrm{U}$ increases.

As already discussed in Section 2, the quantitative values of spin exchanges can be accurately determined by the energy-mapping analysis based on first-principles $\mathrm{DFT}+\mathrm{U}$ or DFT+hybrid calculations. The purpose of Equations (18) and (19) is not to determine the numerical value of any spin exchange, but to show that each spin exchange J consists of two competing components, $\mathrm{J}_{\mathrm{F}}$ and $\mathrm{J}_{\mathrm{AF}}$, that the overall sign of $\mathrm{J}$ is determined by which component dominates, and which electronic parameters govern the strength of each component.

\section{Spin Exchanges Determined by the Ligand p-Orbitals in the Magnetic Orbitals}

To illustrate how spin exchanges of transition metal magnetic ions are controlled by the ligand p-orbitals in their magnetic orbitals, we consider various spin exchanges involving $\mathrm{Cu}^{2+}\left(\mathrm{d}^{9}, \mathrm{~S}=1 / 2\right)$ ions as an example, which typically form axially elongated $\mathrm{CuL}_{6}$ octahedra (Figure 6a). The energies of their d-states are split as $(\mathrm{xz}, \mathrm{yz})<\mathrm{xy}<3 \mathrm{z}^{2}-\mathrm{r}^{2}$ $<\mathrm{x}^{2}-\mathrm{y}^{2}$ (Figure $6 \mathrm{~b}$ ) so that the magnetic orbital of each $\mathrm{Cu}^{2+}$ ion is represented by the $x^{2}-y^{2}$ state, in which the $\mathrm{Cu} \mathrm{x}{ }^{2}-y^{2}$ orbital induces $\sigma$-antibonding with the p-orbitals of four equatorial ligands L (Figure 6c). In this section, we examine how the various types of spin exchanges associated with $\mathrm{Cu}^{2+}$ ions are controlled by the ligand p-orbitals of their $\mathrm{x}^{2}-\mathrm{y}^{2}$ states. The major component of the magnetic orbital of a $\mathrm{Cu}^{2+}$ ion (Figure $6 \mathrm{~d}$ ) is the $\mathrm{Cu}$ d-orbital (i.e., the magnetic orbital "head"), and the minor component the ligand p-orbitals (i.e., the magnetic orbital "tail"). In this section, we probe how the nature and strengths of M-L-M, M-L ... L-M and M-L ... A ... L-M-type exchanges are determined by how the ligand p-orbital tails of their magnetic orbitals are arranged in their exchange paths.

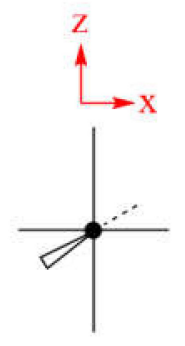

(a)

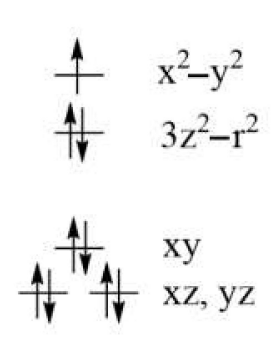

(b)

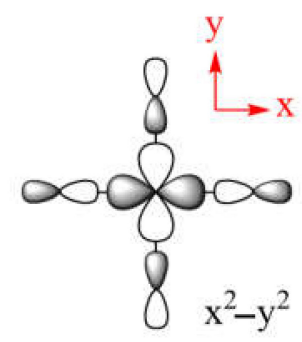

(c)

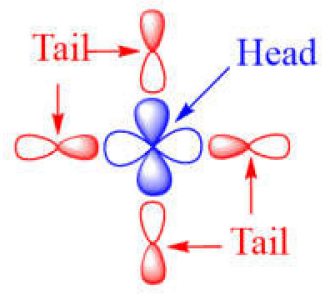

(d)

Figure 6. (a) An axially elongated $\mathrm{CuL}_{6}$ octahedron. (b) The electron configuration of a $\mathrm{Cu}^{2+}\left(\mathrm{d}^{9}\right)$ ion at an axially elongated octahedral site. (c) The magnetic orbital of the $\mathrm{Cu}^{2+}\left(\mathrm{d}^{9}\right)$ ion at an axially elongated octahedral site, which is contained in the $\mathrm{CuL}_{4}$ equatorial plane. (d) The head and tails of the magnetic orbital. 


\section{1. $M-L \ldots L-M$ and $M-L \ldots A \ldots L-M$ Spin Exchanges}

The next-nearest-neighbor (nnn) spin exchange $\mathrm{J}_{\mathrm{nnn}}$ (Figure 7a) that occurs in a $\mathrm{CuL}_{2}$ $\left(\mathrm{L}=\mathrm{O}, \mathrm{Cl}, \mathrm{Br}\right.$ ) ribbon chain, is obtained by sharing the opposite edges of $\mathrm{CuL}_{4}$ square planes. This spin exchange is an example of a strong $\mathrm{Cu}-\mathrm{L} \ldots \mathrm{L}-\mathrm{Cu}$ exchange (Figure $\mathrm{7b}$ ), when the $\mathrm{L}$... L contact distance is in the vicinity of the van der Waals distance. The latter will be assumed to be the case in what follows. The two magnetic orbitals interact across the $\mathrm{L} \ldots$... L contacts through the overlap of their p-orbital tails. This through-space interaction leads to the in-phase and out-of-phase combinations ( $\Psi_{+}$and $\Psi_{-}$, respectively) of the magnetic orbitals (Figure 7c), with energy split $\Delta \mathrm{e}$ between the two (Figure 7d). This makes the AFM component $J_{\mathrm{AF}}$ non-zero. The overlap electron density associated with the interacting p-orbital tails is nearly zero, so the FM component $\mathrm{J}_{\mathrm{F}}$ is practically zero. As a result, $\mathrm{J}_{\mathrm{nnn}}$ becomes AFM.

(a)

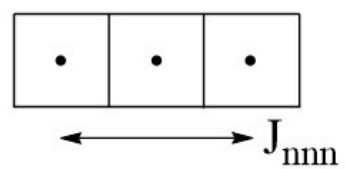

(b)

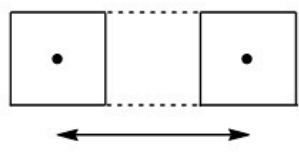

(c)
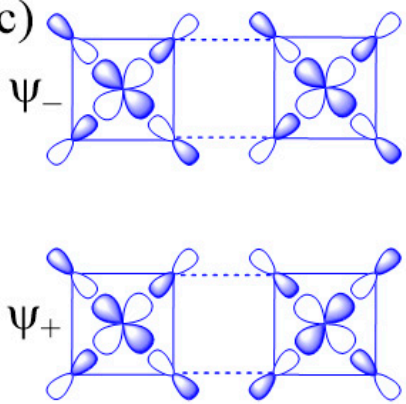

(e)

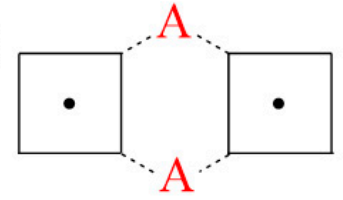

(f)

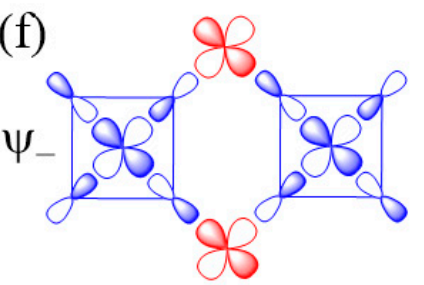

(d)

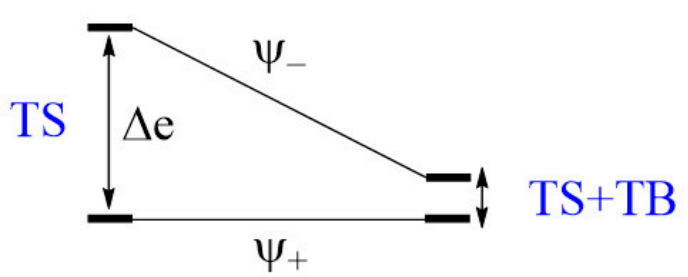

Figure 7. (a) The next-nearest-neighbor spin exchange $\mathrm{J}_{\mathrm{nnn}}$ in a $\mathrm{CuL}_{2}$ ribbon chain made up of edge-sharing $\mathrm{CuL}_{4}$ square planes. (b) A case of strong $\mathrm{Cu}-\mathrm{L}$... L $-\mathrm{Cu}$ exchange, which represents the next-nearest-neighbor exchange $\mathrm{J}_{\text {nnn. }}$ (c) The in-phase and out-of-phase combinations of the two magnetic orbitals, $\Psi_{+}$and $\Psi_{-}$, respectively, associated with the Cu-L ... L-Cu exchange. (d) The large energy split $\Delta \mathrm{e}$ resulting from the through-space (TS) interaction in the Cu-L ... L-Cu exchange becomes small in the $\mathrm{Cu}-\mathrm{L} \ldots \mathrm{A} \ldots \mathrm{L}-\mathrm{Cu}$ exchange as a result of the through-bond (TB) interaction that occurs with the $\Psi_{-}$state. (e) A Cu-L . . A . . . L-Cu exchange generated when each L . . L contact is bridged by a $\mathrm{d}^{0}$ metal cation A. (f) The bonding interaction of the $\Psi_{-}$state with the $d_{\pi}$ orbital of $A$.

In the M-L ... L-M spin exchange $\mathrm{J}_{\text {nnn }}$ discussed above, there are two equivalent exchange paths due to the ribbon structure. Suppose that each L . . . L contact of the M-L ... L-M exchange path is bridged by a $\mathrm{d}^{0}$ transition metal cation $\mathrm{A}$ (e.g., $\mathrm{V}^{5+}$ and $\mathrm{W}^{6+}$ ) to form an $\mathrm{M}-\mathrm{L} \ldots$. . A ... L-M spin exchange (Figure 7e). We now analyze the relative strengths of the M-L ... L-M and M-L ... A ... L-M spin exchanges. Across the L ... L contact of the M-L . . L L-M path, the p-orbital tails of L are combined in-phase in $\Psi_{+}$, but out-of-phase in $\Psi_{-}$. Thus, the empty $\mathrm{d}_{\pi}$ orbital of the cation A interacts in-phase with $\Psi_{-}$(Figure $\left.7 \mathrm{f}\right)$ to lower the energy of $\Psi_{-}$, but it does not interact with $\Psi_{+}$, therefore the energy of $\Psi_{+}$is unaffected. This selective interaction of the bridging $\mathrm{d}^{0}$ cation A with the $\mathrm{L}$... L contact of the M-L ... L-M spin exchange has a dramatic consequence on the strength of an M-L ... A ... L-M spin exchange. When the M-L . . L-M exchange has a strong through-space interaction, the through-bond interaction reduces the large energy split $\Delta \mathrm{e}$ to a small value, thereby weakening the overall $\mathrm{M}-\mathrm{L} \ldots \mathrm{A}$... L-M spin exchange (Figure $7 d$ ). 
Another example of a strong M-L ... L-M exchange occurs when the p-orbital tails of $\mathrm{L}$ are pointed to each other along the $\mathrm{L} \ldots \mathrm{L}$ contact (Figure 8a). The through-space interaction between the magnetic orbitals leads to the in-phase and out-of-phase combinations, $\Psi_{+}$and $\Psi_{-}$, respectively (Figure 8 b), with a large energy split $\Delta$ e between the two (Figure 8c) and a negligible overlap electron density between the interacting p-orbital tails. As a result, the M-L ... L-M spin exchange becomes AFM. If the $\mathrm{L} \ldots \mathrm{L}$ contact of an M-L ... L-M exchange path is bridged by a $\mathrm{d}^{0}$ transition metal cation $\mathrm{A}$ to form an M-L ... A ... L-M spin exchange (Figure 8d), only the $\Psi_{-}$state of the M-L ... L-M path interacts effectively with one of the empty $\mathrm{d}_{\pi}$ orbitals of A (Figure 8e). Thus, a strong through-space interaction in the M-L ... L-M exchange leads to a weak overall M-L ... A ... L-M spin exchange due to the effect of the through-bond interaction (Figure 8c).

(a)

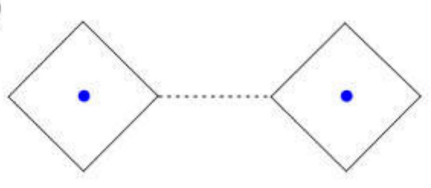

(b)

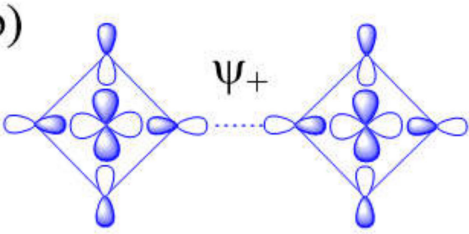

(d)

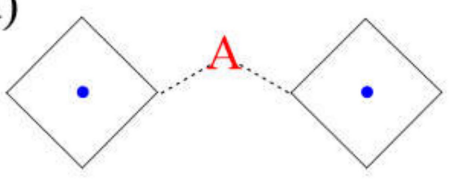

(c)
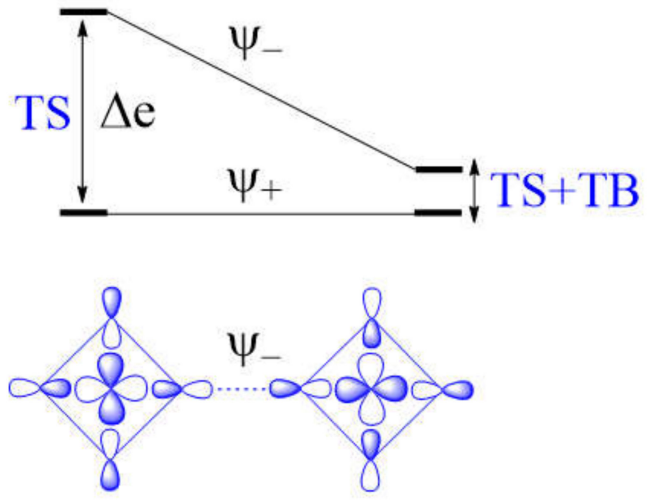

(e)

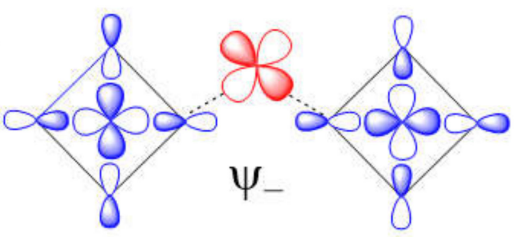

Figure 8. (a) A case of strong $\mathrm{Cu}-\mathrm{L}$... L $-\mathrm{Cu}$ spin exchange where the two $\mathrm{Cu}-\mathrm{L}$ bonds leading to the $\mathrm{L}$... L contacts are linear. (b) The in-phase and out-of-phase combinations ( $\Psi_{+}$and $\Psi_{-}$, respectively) of the two magnetic orbitals, resulting from the through-space (TS) interactions. (c) The energy split $\Delta$ e between $\Psi_{+}$and $\Psi_{-}$is large when the overlap between the p-orbital tails is large. (d) A Cu-L ... A ... L-Cu exchange generated when the $\mathrm{L} \ldots \mathrm{L}$ contact is bridged by a $\mathrm{d}^{0}$ metal cation A. (e) The bonding interaction of the $\Psi_{-}$state with the $\mathrm{d}_{\pi}$ orbital of A. (f) The large energy split $\Delta$ e resulting from the through-space (TS) interaction in the $\mathrm{Cu}-\mathrm{L} \ldots \mathrm{L}-\mathrm{Cu}$ exchange becomes small in the $\mathrm{Cu}-\mathrm{L} \ldots \mathrm{A}$... L-Cu exchange as a result of the through-bond (TB) interaction that occurs primarily with the $\Psi_{-}$state.

An example of very weak $\mathrm{Cu}-\mathrm{L} \ldots \mathrm{L}-\mathrm{Cu}$ exchange is shown in Figure 9a, in which the two magnetic orbitals are arranged such that the p-orbital tails are orthogonal to each other (Figure $9 \mathrm{~b}$ ), and their overlap vanishes so that the energy split between $\Psi_{+}$and $\Psi_{-}$ vanishes (i.e., $\Delta \mathrm{e}=0$ ) (Figure $9 \mathrm{c}$ ) so that $\mathrm{J}_{\mathrm{AF}}=0$. In addition, $\mathrm{J}_{\mathrm{F}}$ should vanish because the overlap electron density resulting from the p-orbital tails will be practically zero. Then, the spin exchange J would be zero. If the $\mathrm{L} \ldots \mathrm{L}$ contact of such an $\mathrm{M}-\mathrm{L} \ldots \mathrm{L}-\mathrm{M}$ exchange path is bridged by a $\mathrm{d}^{0}$ transition metal cation A to form an M-L ... A ... L-M spin exchange (Figure $9 \mathrm{~d}$ ), the $\Psi_{-}$state of the M-L ... L-M path interacts with the empty $\mathrm{d}_{\pi}$ orbital of A, thereby lowering its energy (Figure 9e) while that of the $\Psi_{+}$state is unchanged. Thus, when the M-L ... L-M exchange has a very weak through-space interaction, the through-bond interaction induces the large energy split $\Delta \mathrm{e}$, so that the overall M-L ... A ... L-M spin exchange becomes strong (Figure 9f). 
(a)

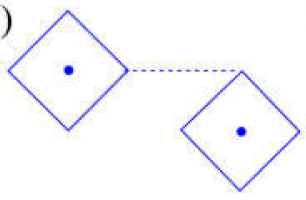

(c)

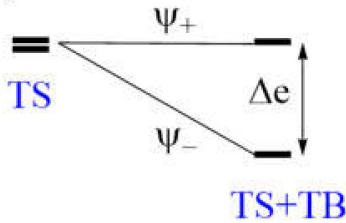

(b)

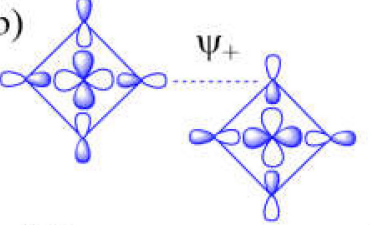

(d)

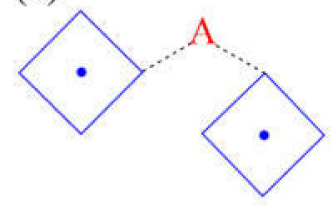

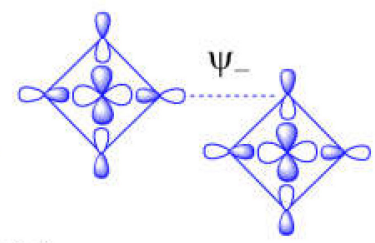

(e)

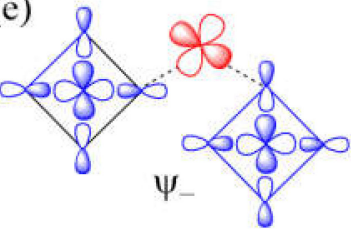

Figure 9. (a) A case of weak $\mathrm{Cu}-\mathrm{L}$... L-Cu spin exchange, where the two $\mathrm{Cu}-\mathrm{L}$ bonds leading to the L ... L contacts are orthogonal. (b) The in-phase and out-of-phase combinations $\left(\Psi_{+}\right.$and $\Psi_{-}$, respectively) of the two magnetic orbitals. (c) The vanishing energy split $\Delta \mathrm{e}$ resulting from the through-space (TS) interaction in the $\mathrm{Cu}-\mathrm{L} \ldots \mathrm{L}-\mathrm{Cu}$ exchange is enhanced in the $\mathrm{Cu}-\mathrm{L} \ldots \mathrm{A}$... $\mathrm{L}-\mathrm{Cu}$ exchange as a result of the through-bond (TB) interaction that occurs primarily with the $\Psi_{-}$ state. (d) A Cu-L ... A ... L-Cu exchange generated when the L . . L contact is bridged by a d $\mathrm{d}^{0}$ metal cation A. (e) The in-phase interaction of the $\Psi_{-}$state with the $\mathrm{d}_{\pi}$ orbital of A.

In short, a strong M-L ... L-M exchange becomes a weak M-L ... A ... L-M exchange when the $\mathrm{L} \ldots$. L linkage is bridged by $\mathrm{d}^{0}$ cations, while a weak $\mathrm{M}-\mathrm{L} \ldots \mathrm{L}-\mathrm{M}$ exchange becomes a strong $\mathrm{M}-\mathrm{L} \ldots$. A . . L-M exchange when the $\mathrm{L} \ldots \mathrm{L}$ linkage is bridged by a $\mathrm{d}^{0}$ cation.

\section{2. $M-L-M$ Spin Exchanges}

The Goodenough-Kanamori rules cover these types of spin exchanges [5-9]. For the sake of completeness, we discuss these types of spin exchanges from the viewpoint of the ligand p-orbital tails on the basis of Equations (18) and (19). Let us consider a $\mathrm{Cu}_{2} \mathrm{~L}_{6}$ dimer resulting from two $\mathrm{CuL}_{4}$ square planes obtained by sharing an edge (Figure 10a), where the ligand $\mathrm{L}$ can be $\mathrm{O}, \mathrm{Cl}$, or $\mathrm{Br}$. The two magnetic orbitals associated with the nearest-neighbor $(\mathrm{nn})$ spin exchange $\mathrm{J}_{\mathrm{nn}}$, presented in Figure $10 \mathrm{~b}$, interact at the bridging ligands $\mathrm{L}$ of the $\mathrm{M}-\mathrm{L}-\mathrm{M}$ paths. If the $\mathrm{CuL}_{4}$ units have an ideal square planar shape, the $\angle \mathrm{M}-\mathrm{L}-\mathrm{M}$ angle becomes $90^{\circ}$ so that the two p-orbital tails at the bridging ligands $\mathrm{L}$ are orthogonal to each other. Thus, as discussed in Section 3, the overlap integral between them is zero. Therefore, the in-phase and out-of-phase combinations of the two magnetic orbitals (Figure 10b) is not split in energy, so $\Delta \mathrm{e}=0$ (Figure 10c) and $\mathrm{J}_{\mathrm{AF}}=0$. However, the overlap electron density between the two p-orbital tails at the bridging ligand $\mathrm{L}$ is not zero, i.e., $\mathrm{J}_{\mathrm{F}}>0$. Thus, the $\mathrm{M}-\mathrm{L}-\mathrm{M}$ spin exchange becomes FM. When the $\angle \mathrm{M}-\mathrm{L}-\mathrm{M}$ angle deviates from $90^{\circ}$ (Figure 10d), the two p-orbital tails at the bridging ligands $\mathrm{L}$ are no longer orthogonal to each other so the overlap integral between them is non-zero. Therefore, the in-phase and out-of-phase combinations of the two magnetic orbitals (Figure 10e) differ in energy, so $\Delta \mathrm{e}>0$ (Figure 10f) and $\mathrm{J}_{\mathrm{AF}}$ is non-zero. The overlap electron density between the two $\mathrm{p}$-orbital tails is non-zero, so $\mathrm{J}_{\mathrm{F}}$ is non-zero. Thus, whether the spin exchange is FM or AFM depends on which component, $\mathrm{J}_{\mathrm{F}}$ or $\mathrm{J}_{\mathrm{AF}}$, dominates, which in turn depends on the $\angle \mathrm{M}-\mathrm{L}-\mathrm{M}$ angle $\phi$. Typically, the angle $\phi$ where FM changes to AFM is slightly greater than $90^{\circ}$ due to the involvement of the ligand s-orbital [15], which is commonly neglected for simplicity. 

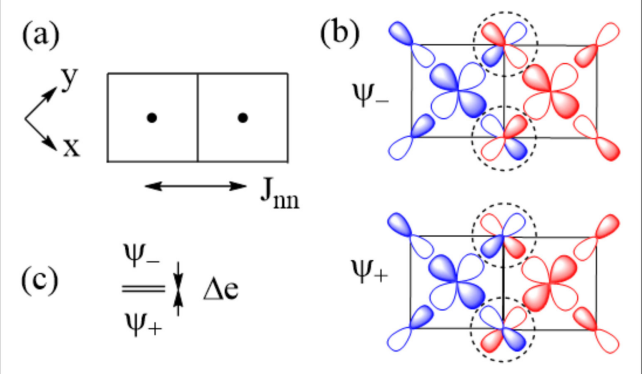

(d)

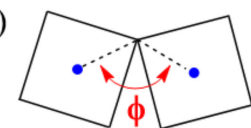

(e)

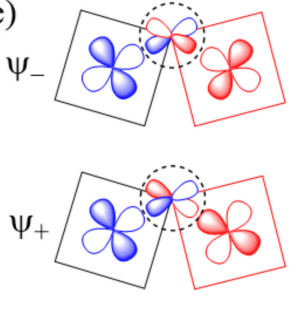

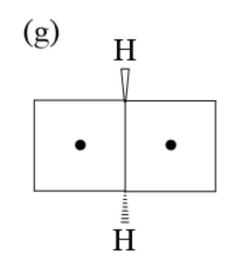

(h)

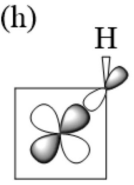

(i)

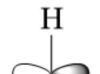

(j)

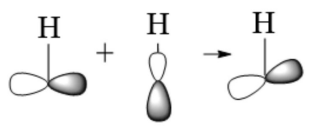

(k)

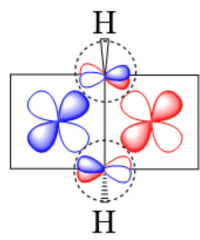

Figure 10. (a-c) A Cu-L-Cu spin exchange with $\angle \mathrm{M}-\mathrm{L}-\mathrm{M}$ angle $\phi=90^{\circ}$ : This occurs in $\mathrm{Cu}_{2} \mathrm{~L}_{6}$ dimer shown in (a), which is made up of two coplanar $\mathrm{CuL}_{4}$ square planes by sharing an edge. The in-phase and out-of-phase combinations of the two magnetic orbitals in (b), and the energy split $\Delta \mathrm{e}$ between the two in (c). (d-f) A Cu-L-Cu spin exchange with $\angle \mathrm{M}-\mathrm{L}-\mathrm{M}$ angle $\phi>90^{\circ}$ : This occurs when two non-coplanar $\mathrm{CuL}_{4}$ square planes are corner-shared as in (d). The in-phase and out-of-phase combinations of the two magnetic orbitals in (e), and the energy split $\Delta$ e between the two in (f). (g-k) Effect of the molecular anions $\mathrm{OH}^{-}$on the spin exchange of the edge-sharing $\mathrm{Cu}_{2} \mathrm{O}_{6}$ dimer: the shared edge consists of two $\mathrm{OH}^{-}$ ions in (g); the p-orbital tail of one $\mathrm{OH}^{-}$ligand in $\mathrm{CuO}_{4}$ square plane expected if the $\mathrm{OH}^{-}$is treated as $\mathrm{O}^{2-}$ in $(\mathbf{h})$; the three oxygen lone pairs associated with an isolated $\mathrm{OH}^{-}$in (i); the tilting of the $\mathrm{O} 2 \mathrm{p}$-orbital tail by mixing two oxygen lone pairs in (j); and the arrangement of the tilted $\mathrm{O} 2 \mathrm{p}$-tails at the bridging $\mathrm{O}$ atoms of the $\mathrm{Cu}_{2} \mathrm{O}_{6}$ dimer in (k).

So far in our discussion, it has been implicitly assumed that each main-group ligand $\mathrm{L}$ exists as a spherical anion (e.g., each $\mathrm{O}$ as an $\mathrm{O}^{2-}$ anion, and each $\mathrm{Cl}$ atom as a $\mathrm{Cl}^{-}$ anion). However, this picture is not quite accurate when the ligand atom makes a strong covalent bonding with another main-group element to form a molecular anion such as $\mathrm{OH}^{-}$. Suppose that each $\mathrm{O}$ atom on the shared edge of the $\mathrm{CuO}_{6}(\mathrm{~L}=\mathrm{O})$ dimer is not a $\mathrm{O}^{2-}$ but an $\mathrm{OH}^{-}$anion (Figure $10 \mathrm{~g}$ ). Then, the ligand p-orbital tail on that $\mathrm{O}$ cannot be the p-orbital pointed along one lobe of the $\mathrm{Cu} \mathrm{x}^{2}-\mathrm{y}^{2}$ orbital (Figure 10h) because it is incompatible with the $\mathrm{O}-\mathrm{H}$ bonding, which has three directional $\mathrm{O}$ lone pairs depicted in Figure 10i. To satisfy both the strong covalent-bonding with $\mathrm{H}$ and the weak covalentbonding with $\mathrm{Cu}$, the $\mathrm{O}$ lone pair of $\mathrm{OH}^{-}$tilts slightly toward one lobe of the $\mathrm{x}^{2}-\mathrm{y}^{2}$ orbital (Figure 10j). As a result, the ligand p-orbital tails, arising from the two magnetic orbitals at the bridging $\mathrm{O}$ atoms, are not orthogonal as in Figure 10b but become more parallel to each other (Figure 10k). As a result, the spin exchange between the two $\mathrm{Cu}^{2+}$ ions in Figure 10g becomes AFM (see below). Another molecular anion of interest is the carbonate ion $\mathrm{CO}_{3}{ }^{2-}$, in which each $\mathrm{O}$ atom makes a strong covalent bond with $\mathrm{C}$; therefore, the $\mathrm{O}$ atoms of $\mathrm{CO}_{3}{ }^{2-}$ should not be treated as isolated $\mathrm{O}^{2-}$ anions in their coordination with transition metal cations. In general, the presence of molecular anions such as $\mathrm{OH}^{-}$and $\mathrm{CO}_{3}{ }^{2-}$ in a magnetic solid makes it difficult to deduce, on a qualitative reasoning, what its spin lattice would be. This is where the quantitative energy-mapping analysis is indispensable, because it does not require any qualitative reasoning.

\subsection{Qualitative Rules for Spin Exchanges Based on the p-Orbital Tails of Magnetic Orbitals}

In a magnetic orbital of an $\mathrm{ML}_{\mathrm{n}}$ polyhedron, the $\mathrm{d}$-orbital of $\mathrm{M}$ dictates by its symmetry which p-orbitals of the ligands $\mathrm{L}$ become the p-orbital tails. From the viewpoint of orbital interaction, a spin exchange between magnetic ion is none other than the interaction 
between their magnetic orbitals. The latter is caused by the interaction between their p-orbital tails, not by that between their d-orbital heads. In other words, a spin exchange is not a "head-to-head" interaction but a "tail-to-tail" interaction. By considering these tail-to-tail interactions described above, we arrive at the following four qualitative rules governing the nature and strengths of the $\mathrm{M}-\mathrm{L} \ldots \mathrm{L}-\mathrm{M}$ and $\mathrm{M}-\mathrm{L} \ldots \mathrm{A}$... L-M type exchanges under the assumption that the $\mathrm{L} \ldots \mathrm{L}$ contact distance is in the vicinity of the van der Waals distance:

(1) When the p-orbital tails generate a large overlap integral but a small overlap electron density, the M-L ... L-M exchange is AFM.

(2) When the p-orbital tails generate a small overlap integral but a large overlap electron density, the $\mathrm{M}-\mathrm{L}$... L-M exchange is FM.

(3) When the p-orbital tails generate neither a non-zero overlap integral nor a non-zero overlap electron density, the $\mathrm{M}-\mathrm{L}$... L-M exchange vanishes.

(4) When the $\mathrm{M}-\mathrm{L}$. . . L-M exchange is strongly AFM, the corresponding M-L . . A ... $\mathrm{L}-\mathrm{M}$ becomes a weak exchange. When the $\mathrm{M}-\mathrm{L}$... L-M exchange is a weak exchange, the corresponding $\mathrm{M}-\mathrm{L} \ldots \mathrm{A} \ldots \mathrm{L}-\mathrm{M}$ becomes strongly AFM.

These rules on the M-L .. L L-M and M-L . . A . . L L-M exchanges should be used together with the Goodenough-Kanamori rules in choosing a proper set of spin exchanges to evaluate using the energy-mapping analysis based on DFT+U or DFT+hybrid calculations. In principle, this analysis can provide quantitative values for any possible exchanges of a given magnetic system. However, even this quantitative tool cannot determine the value of any spin exchange unless it is included in the set of spin exchanges for the energymapping analysis. It is paramount to consider in detail the structural features governing the strengths of spin exchanges in order not to miss exchange paths crucial for defining the correct spin lattice of a given solid.

\section{Representative Examples}

In Section 4, we analyzed the structural features governing the nature of the three types of spin exchanges, i.e., $\mathrm{M}-\mathrm{L}-\mathrm{M}, \mathrm{M}-\mathrm{L}$... L-M and $\mathrm{M}-\mathrm{L}$. . . A ... L-M, which occur in various magnetic solids. This section will discuss the occurrence of these exchanges in actual magnetic solids by analyzing the crystal structures and magnetic properties of four representative magnetic solids, $\alpha-\mathrm{CuV}_{2} \mathrm{O}_{6}, \mathrm{LiCuVO}_{4},(\mathrm{CuCl}) \mathrm{LaNb}_{2} \mathrm{O}_{7}$ and $\mathrm{Cu}_{3}\left(\mathrm{CO}_{3}\right)_{3}(\mathrm{OH})_{3}$. $\alpha-\mathrm{CuV}_{2} \mathrm{O}_{6}, \mathrm{LiCuVO}_{4}$, and $(\mathrm{CuCl}) \mathrm{LaNb}_{2} \mathrm{O}_{7}$ were chosen to show that correct spin lattices can be readily predicted by the qualitative rules of Section 4.3, although they have to be confirmed by performing the energy-mapping analyses. Azurite $\mathrm{Cu}_{3}\left(\mathrm{CO}_{3}\right)_{2}(\mathrm{OH})_{2}$ was chosen to demonstrate that the spin lattice of a certain magnetic system cannot be convincingly deduced solely on the basis of the qualitative rules. The magnetic ions of such a system are coordinated with molecular anions in which the first-coordinate main-group ligands $\mathrm{L}$ make strong covalent bonds with other main-group elements (e.g., $\mathrm{H}$ in the $\mathrm{OH}^{-}$ion, and $\mathrm{C}$ in the $\mathrm{CO}_{3}{ }^{2-}$ ion). In such a case, use of the energy-mapping analysis is the only recourse with which to find the spin lattice correct for a given system.

\subsection{Two-Dimensional Behavior of $\alpha-\mathrm{CuV}_{2} \mathrm{O}_{6}$}

The magnetic properties of $\alpha-\mathrm{CuV}_{2} \mathrm{O}_{6}$ were initially analyzed in terms of a onedimensional (1D) spin $S=1 / 2$ Heisenberg chain model with uniform nearest-neighbor AFM spin exchange [16-18]. However, a rather high Néel temperature of $\sim 22.4 \mathrm{~K}$ indicated the occurrence of a substantial interchain spin exchange of the order of $50 \%$ of the intrachain exchange, casting serious doubts on the applicability of a simple chain description. $\alpha-\mathrm{CuV}_{2} \mathrm{O}_{6}$ consists of $\mathrm{CuO}_{4}$ chains, made up of edge-sharing $\mathrm{CoO}_{6}$ octahedra, which run along the a-direction (Figure 11a). If the two axially elongated $\mathrm{Cu}-\mathrm{O}$ bonds are removed from each $\mathrm{CuO}_{6}$ octahedron to identify its $\mathrm{CuO}_{4}$ equatorial plane containing the magnetic orbital, one finds that each $\mathrm{CuO}_{4}$ chain of edge-sharing $\mathrm{CoO}_{6}$ octahedra becomes a chain of stacked $\mathrm{CuO}_{4}$ square planes (Figure 11b). In each stack-chain along the a-direction, adjacent $\mathrm{CuO}_{4}$ square planes are parallel to each other such that the adjacent magnetic 
orbitals generate neither a non-zero overlap nor a non-zero overlap electron density. The same is true between adjacent $\mathrm{CuO}_{4}$ square planes along the b-direction (Figures $11 \mathrm{c}$ and 12a). However, between adjacent $\mathrm{CuO}_{4}$ square planes along the c-direction, an almost linear $\mathrm{Cu}-\mathrm{O}$... O $-\mathrm{Cu}$ contact occurs with $\mathrm{O}$... O distance of $2.757 \AA$ (Figures 11c and $12 \mathrm{~b})$, slightly shorter than the van der Waals distance of $2.80 \AA$ A. Thus, this $\mathrm{Cu}-\mathrm{O} \ldots \mathrm{O}-\mathrm{Cu}$ spin exchange along the c-direction $\left(\mathrm{J}_{\mathrm{c}}\right)$ should be substantial.

(a)

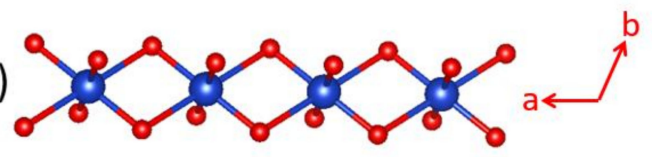

(b)<smiles></smiles>

(d)

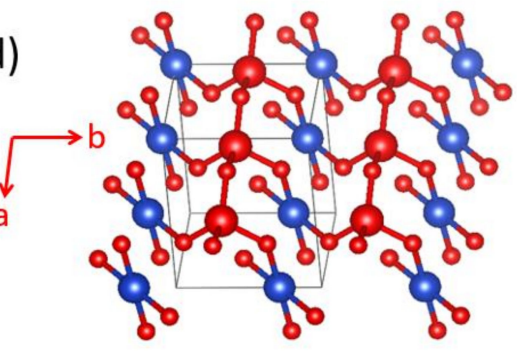

(c)

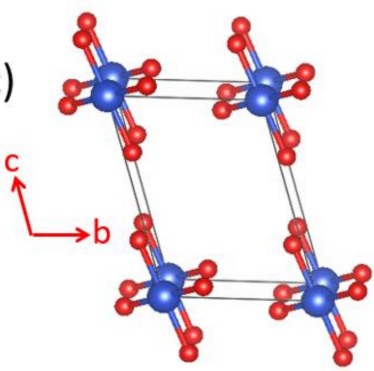

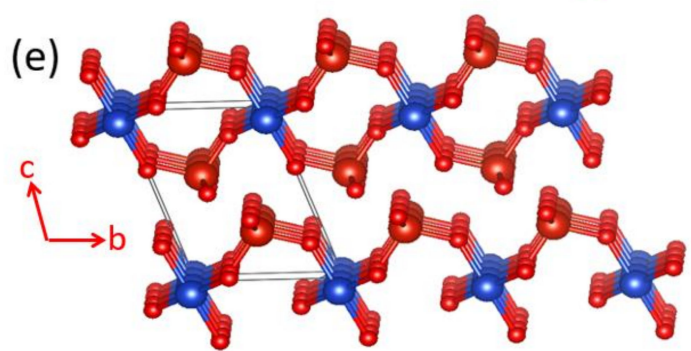

Figure 11. Crystal structure of $\alpha-\mathrm{CuV}_{2} \mathrm{O}_{6}$, where the blue spheres represent the $\mathrm{Cu}$ atoms, and the large and small red spheres the $\mathrm{V}$ and $\mathrm{O}$ atoms, respectively: (a) $\mathrm{A} \mathrm{CuO}_{4}$ chain along the a-direction, which is made up of edge-sharing, axially-elongated, $\mathrm{CuO}_{6}$ octahedra. (b) A stack of $\mathrm{CuO}_{4}$ square planes along the a-direction, which results from the chain of edge-sharing $\mathrm{CuO}_{6}$ octahedra by removing the axial $\mathrm{Cu}-\mathrm{O}$ bonds. (c) Stacks of $\mathrm{CuO}_{4}$ square planes running along the c-direction. (d) One sheet of $\mathrm{CuO}_{4}$ stack chains parallel to the $\mathrm{a}-\mathrm{b}$ plane condensed with chains of corner-sharing $\mathrm{VO}_{4}$ tetrahedra on one side of the sheet. (e) Stacking of $\mathrm{CuV}_{2} \mathrm{O}_{6}$ layers forming $\alpha-\mathrm{CuV}_{2} \mathrm{O}_{6}$.
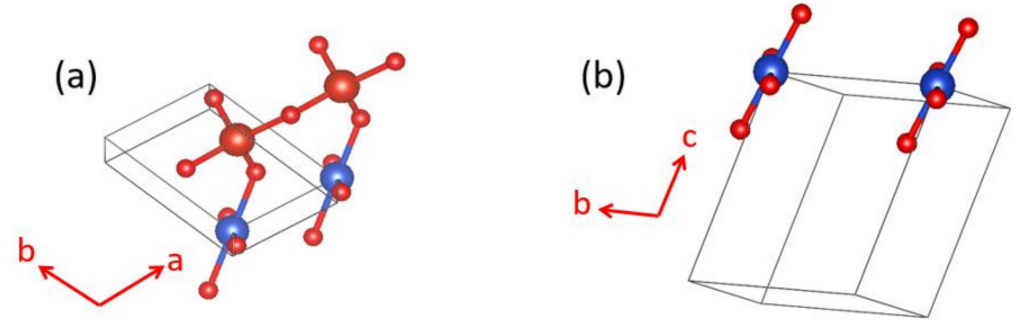

(c)

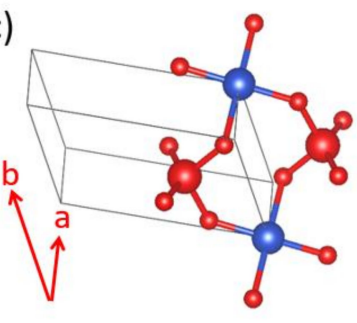

(d)

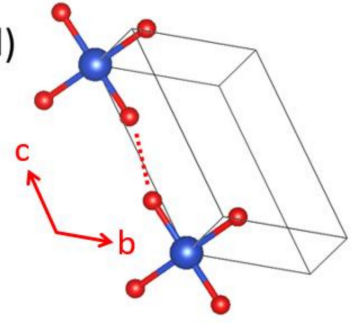

(e)

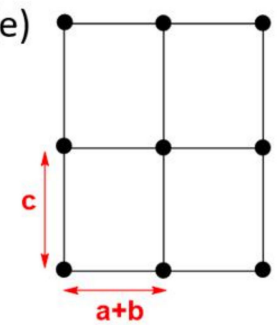

Figure 12. Spin exchange paths and spin lattice of $\alpha-\mathrm{CuV}_{2} \mathrm{O}_{6}$ : (a) Spin exchange along the b-direction, $\mathrm{J}_{\mathrm{b}}$; (b) Spin exchange along the c-direction, $\mathrm{J}_{\mathrm{c}}$; (c) Spin exchange along the a-direction, $\mathrm{J}_{\mathrm{a}}$; (d) Spin exchange along the $(\mathrm{a}+\mathrm{b})$-direction, $\mathrm{J}_{\mathrm{a}+\mathrm{b}} ;(\mathbf{e})$ Rectangular spin lattice made up of $\mathrm{J}_{\mathrm{c}}$ and $\mathrm{J}_{\mathrm{a}+\mathrm{b}}$. 
Each sheet of $\mathrm{CuO}_{4}$ stack-chains parallel to the $\mathrm{a}-\mathrm{b}$ plane is corner-shared with $\mathrm{V}_{2} \mathrm{O}_{6}$ chains, above and below the sheet (Figure 11d), to form layers of composition $\mathrm{CuV}_{2} \mathrm{O}_{6}$ (Figure 11e). Each $\mathrm{V}_{2} \mathrm{O}_{6}$ chain is made up of corner-sharing $\mathrm{VO}_{4}$ tetrahedra containing $\mathrm{V}^{5+}$ $\left(\mathrm{d}^{0}, \mathrm{~S}=0\right)$ ions. As a consequence, adjacent $\mathrm{CuO}_{4}$ square planes are corner-shared with $\mathrm{V}_{2} \mathrm{O}_{6}$ chains (Figure 12c). The adjacent $\mathrm{CuO}_{4}$ square planes are not bridged by a single $\mathrm{VO}_{4}$ tetrahedron; therefore, the spin exchange along the a-direction, $\mathrm{J}_{\mathrm{a}}$, is expected to be weak. Along the $(\mathrm{a}+\mathrm{b})$-direction, two adjacent $\mathrm{CuO}_{4}$ square planes are bridged by $\mathrm{VO}_{4}$ to form two $\mathrm{Cu}-\mathrm{O} \ldots \mathrm{V}^{5+} \ldots \mathrm{O}-\mathrm{Cu}$ paths, in which the two $\mathrm{Cu}-\mathrm{O}$ bonds in each path have a near-orthogonal arrangement.

As already discussed (Figure 10), this $\mathrm{Cu}-\mathrm{O} \ldots \mathrm{V}^{5+} \ldots \mathrm{O}-\mathrm{Cu}$ spin exchange should be substantial due to the through-bond effect of the $\mathrm{V}^{5+}$ cation. Consequently, the spin lattice of $\alpha-\mathrm{CuV}_{2} \mathrm{O}_{6}$ must be described by a two-dimensional (2D) rectangular lattice defined by $\mathrm{J}_{\mathrm{a}+\mathrm{b}}$ and $\mathrm{J}_{\mathrm{c}}$. In support of this analysis, the energy-mapping analysis based on DFT+U calculations with $\mathrm{U}_{\text {eff }}=4 \mathrm{eV}$ show that $\mathrm{J}_{\mathrm{a}+\mathrm{b}}$ and $\mathrm{J}_{\mathrm{c}}$ are the two dominant spin exchanges, and are nearly equal in magnitude, namely, $\mathrm{J}_{\mathrm{a}+\mathrm{b}}=86.8 \mathrm{~K}$ and $\mathrm{J}_{\mathrm{c}} / \mathrm{J}_{\mathrm{a}+\mathrm{b}}=0.88$ [19]. In agreement with this finding, one re-investigation of the magnetic properties of $\alpha-\mathrm{CuV}_{2} \mathrm{O}_{6}$ clearly attested a $2 \mathrm{D} \mathrm{S}=1 / 2$ rectangular spin lattice model with an anisotropy ratio of 0.7 [19]. The magnetic structure determined from neutron powder diffraction data was in best agreement with these $\mathrm{DFT}+\mathrm{U}$ calculations. In terms of chemical bonding, $\alpha-\mathrm{CuV}_{2} \mathrm{O}_{6}$ consists of $\mathrm{CuV}_{2} \mathrm{O}_{6}$ layers stacked along the c-direction. There is no chemical bonding between adjacent layers; only van der Waals interactions. In terms of magnetic bonding, however, $\alpha-\mathrm{CuV}_{2} \mathrm{O}_{6}$ consists of $2 \mathrm{D}$ spin lattices parallel to the $(\mathrm{a}+\mathrm{b})-\mathrm{c}$ plane. There is negligible magnetic bonding perpendicular to this plane.

\subsection{One-Dimensional Chain Behavior of $\mathrm{LiCuVO}_{4}$}

$\mathrm{LiCuVO}_{4}$ consists of axially elongated $\mathrm{CuO}_{6}$ octahedra, which form edge-sharing $\mathrm{CuO}_{4}$ chains along the b-direction, which are corner-shared with $\mathrm{VO}_{4}$ tetrahedra containing $\mathrm{V}^{5+}\left(\mathrm{d}^{0}, \mathrm{~S}=0\right)$ ions (Figure 13a). When the axial $\mathrm{Cu}-\mathrm{O}$ bonds are deleted, one finds $\mathrm{CuVO}_{4}$ layers in which the $\mathrm{CuO}_{2}$ ribbon chains are corner-shared by $\mathrm{VO}_{4}$ tetrahedra (Figure 13b). A perspective view of a single $\mathrm{CuVO}_{4}$ layer approximately along the c-direction (Figure 13c) shows that each $\mathrm{VO}_{4}$ tetrahedron bridges two neighboring $\mathrm{CuO}_{2}$ ribbon chains. Thus, the spin exchanges of interest are the nearest-neighbor exchanges $\mathrm{J}_{\mathrm{nn}}$ of the $\mathrm{Cu}-\mathrm{O}-\mathrm{Cu}$ type and the next-nearest-neighbor spin exchange $\mathrm{J}_{\text {nnn }}$ of the $\mathrm{Cu}-\mathrm{O} \ldots \mathrm{O}-\mathrm{Cu}$ type within each $\mathrm{CuO}_{2}$ ribbon chain as well as the interchain spin exchange $\mathrm{J}_{\mathrm{a}}$ along the a-direction of the $\mathrm{Cu}-\mathrm{O} \ldots \mathrm{V}^{5+} \ldots$ O-Cu type (Figure $13 \mathrm{~d}$ ).

In the absence of the $\mathrm{V}^{5+}$ ion, the exchange $\mathrm{J}_{\mathrm{a}}$ would be similar in strength to $\mathrm{J}_{\mathrm{nnn}}$. However, the $\mathrm{O} \ldots \mathrm{V}^{5+} \ldots \mathrm{O}$ bridges will weaken the strength of $\mathrm{J}_{\mathrm{a}}$, as discussed in Figure 7. Furthermore, there is no spin exchange path between adjacent $\mathrm{CuVO}_{4}$ layers. Consequently, the spin lattice of $\mathrm{LiCuVO}_{4}$ is a $1 \mathrm{D}$ chain running along the b-direction, as is the 1D ribbon chain. The major cause for the occurrence of the 1D chain character is the $\mathrm{Cu}-\mathrm{O} \ldots \mathrm{V}^{5+} \ldots \mathrm{O}-\mathrm{Cu}$ spin exchange, which nearly vanishes because the effect of the through-space interaction is canceled by that of the through-bond interaction. In agreement with this reasoning, DFT+U calculations with $\mathrm{U}_{\text {eff }}=4 \mathrm{eV}$ show that $\mathrm{J}_{\mathrm{nnn}}$ is strongly AFM (i.e., $208.7 \mathrm{~K}$ ), while $\mathrm{J}_{\mathrm{nn}}$ and $\mathrm{J}_{\mathrm{a}}$ are weakly FM (i.e., $\mathrm{J}_{\mathrm{nn}} / \mathrm{J}_{\mathrm{nnn}}=-0.12$, and $\left.\mathrm{Ja}_{\mathrm{a}} / \mathrm{J}_{\mathrm{nnn}}=-0.08\right)[20]$.

As in the case of $\alpha-\mathrm{CuV}_{2} \mathrm{O}_{6}$, the magnetic properties of $\mathrm{LiCuVO}_{4}$ were initially analyzed in terms of a 1D chain with uniform nearest-neighbor Heisenberg spin exchange [21-23]. The magnetic susceptibility of $\mathrm{LiCuVO}_{4}$ showed a typical broad maximum at about $28 \mathrm{~K}$, characteristic for $1 \mathrm{D}$ behavior with strong intrachain spin exchanges, whereas long-range AFM ordering was detected only below $\sim 2.2 \mathrm{~K}$. The need to modify this simple 1D description was brought about by Gibson et al., who determined the magnetic structure of $\mathrm{LiCuVO}_{4}$ from single crystal neutron diffraction [24]. They found that the spins of each $\mathrm{CuO}_{2}$ ribbon chain had a cycloid structure (Figure $14 \mathrm{a}$ ) with adjacent $\mathrm{Cu}^{2+}$ moments making an angle of slightly less than $90^{\circ}$. The incommensurate cycloid structure is ex- 
plained by spin frustration due to competing exchange $\mathrm{J}_{\mathrm{nn}}$, which is $\mathrm{FM}$, and $\mathrm{J}_{\mathrm{nnn}}$, which is $\mathrm{AFM}[20,24-26]$. If all the spins of $\mathrm{a} \mathrm{CuO}_{2}$ ribbon chain were to be collinear, the ribbon chain cannot satisfy all nearest-neighbor exchanges FM and all next-nearest-neighbors AFM simultaneously. Thus, the spin arrangement of the $\mathrm{CuO}_{2}$ ribbon chain is spin-frustrated. To reduce the extent of this spin frustration, the spins of the ribbon chain adopt a noncollinear spin arrangement. The noncollinear spin arrangement observed for $\mathrm{LiCuVO}_{4}$ has a cycloid structure in each ribbon chain, in which the nearest-neighbor spins are nearly orthogonal to each other, while the next-nearest-neighbor spins generate a near-AFM arrangement (Figure 14a) [24,25]. In a cycloid, each successive spin in the $\mathrm{CuO}_{2}$ ribbon chain rotates in one direction by a certain angle. Thus, a cycloid structure is chiral in nature, which means that the alternative cycloid structure opposite in chirality but identical in energy is equally probable (Figure 14b) [27].
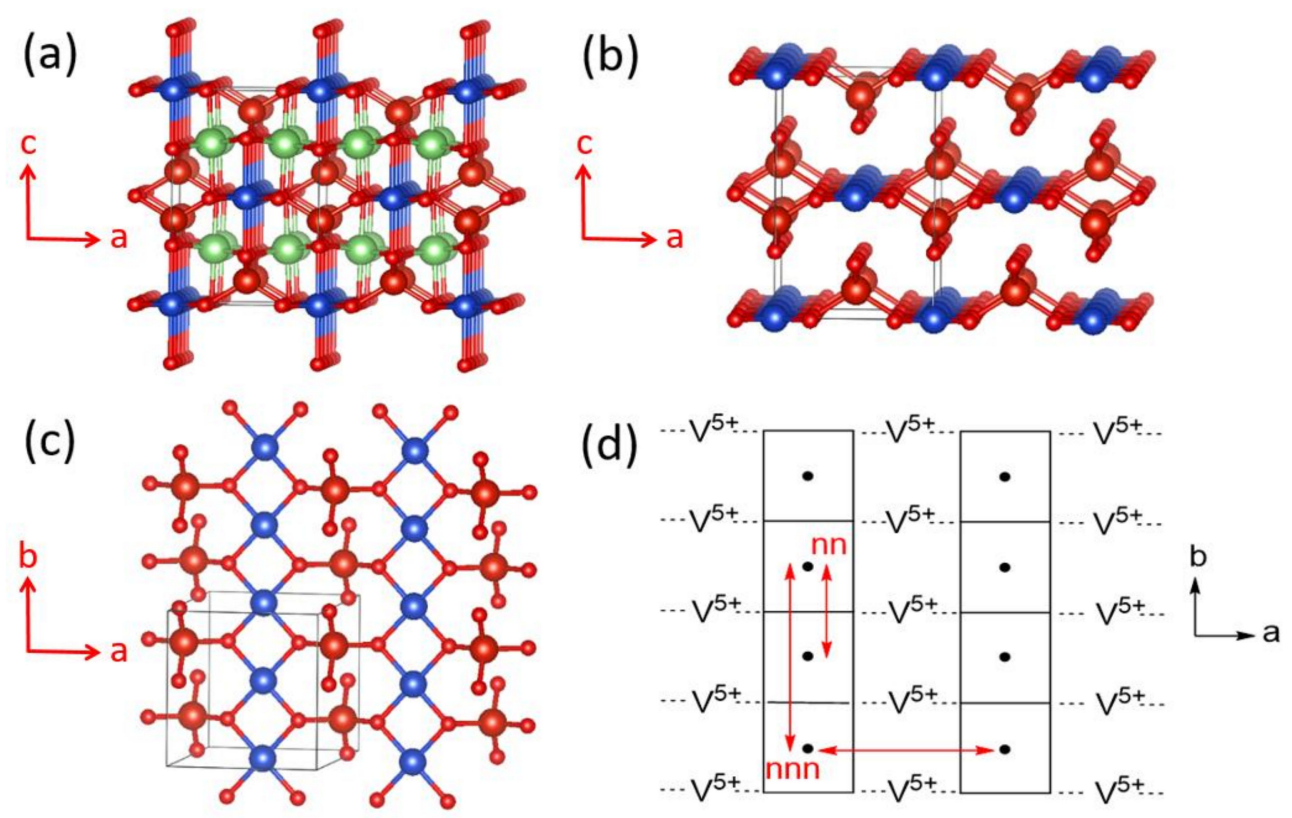

Figure 13. (a) The crystal structure of $\mathrm{LiCuVO}_{4}$ viewed approximately along the b-direction, where the blue and green spheres represent the $\mathrm{Cu}$ and $\mathrm{Li}$ atoms, respectively, and the large and small red spheres represent the $\mathrm{V}$ and $\mathrm{O}$ atoms, respectively. (b) The $\mathrm{CuVO}_{4}$ lattice resulting from $\mathrm{LiCuVO}_{4}$ by removing the axial $\mathrm{Cu}-\mathrm{O}$ bonds and $\mathrm{Li}$ atoms. (c) A perspective view of a single $\mathrm{CuVO}_{4}$ layer approximately along the c-direction. (d) The spin exchange paths present in a single $\mathrm{CuVO}_{4}$ layer, where the labels $\mathrm{nn}$, nnn and a represent the spin exchanges $\mathrm{J}_{\mathrm{nn}}, \mathrm{J}_{\mathrm{nnn}}$ and $\mathrm{J}_{\mathrm{a}}$, respectively.

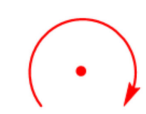

(a)

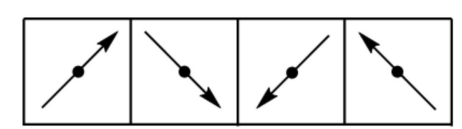

(b)

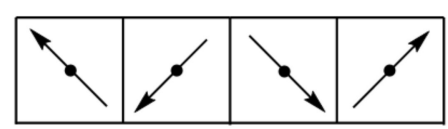

Figure 14. (a,b) Two cycloids, which are opposite in chirality but are identical in energy.

In general, when the temperature is lowered below a certain temperature, $\mathrm{T}_{\mathrm{SDW}}$, a moderately spin-frustrated magnetic system gives rise to two cycloids of opposite chirality with equal probability. The resulting superposition of the two (Figure 15a-c) leads to a state known as a spin density wave (SDW) $[27,28]$. The latter becomes transverse if the preferred spin orientation at each magnetic ion is perpendicular to the SDW propagation direction, but becomes longitudinal if the spin orientation prefers the SDW propagation direction 
(Figure $15 \mathrm{~d}-\mathrm{f}$ ). When the temperature is lowered further below $\mathrm{T}_{\mathrm{SDW}}$, the electronic structure of the spin-lattice may relax to energetically favor one of the two chiral cycloids so that one can observe a cycloid state at a temperature slightly below $\mathrm{T}_{\mathrm{SDW}}$. The repeat unit of a cycloid is determined by the spin frustration present in the magnetic system, therefore a cycloid phase is typically incommensurate.
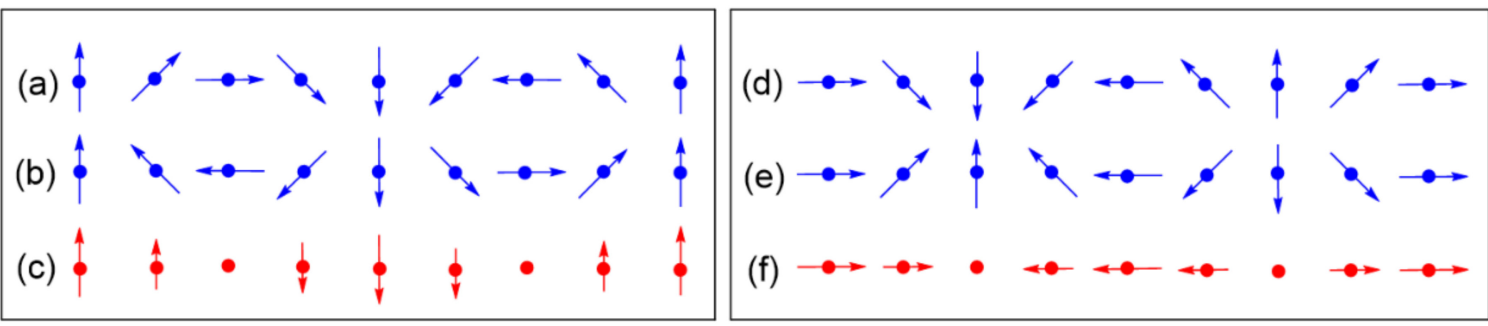

Figure 15. The superposition of the two chiral cycloids in (a); (b) leads to the transverse SDW in (c). The superposition of the two chiral cycloids in (d); (e) leads to the longitudinal SDW in (f). For ease of illustration, the angle of the spin rotation was taken to be $45^{\circ}$.

In the cycloid state, $\mathrm{LiCuVO}_{4}$ exhibits ferroelectricity [29-31], because a cycloid structure lacks inversion symmetry. The polarization of $\mathrm{LiCuVO}_{4}$ can be switched with magnetic and electric fields [32-35]. $\mathrm{LiCuVO}_{4}$ has attracted special attention for the possibility of inducing new phases by applying external magnetic fields. Saturation of the $\mathrm{Cu}$ moments occurs above $\sim 45 \mathrm{~T}$, depending on the orientation of the crystal [36]. The competition between $\mathrm{Jnn}_{\mathrm{nn}}$ and $\mathrm{J}_{\mathrm{nnn}}$ in $\mathrm{LiCuVO}_{4}$ is considered a promising setting for unusual bond nematicity and spin nematic phases close to the full magnetic saturation [37-45].

\subsection{Spin Gap Behavior from the $\mathrm{CuO}_{2} \mathrm{Cl}_{2}$ Perovskite Layer of (CuCl) LaNb${ }_{2} \mathrm{O}_{7}$}

$(\mathrm{CuCl}) \mathrm{LaNb}_{2} \mathrm{O}_{7}$ consists of $\mathrm{CuClO}_{2}$ and $\mathrm{LaNb}_{2} \mathrm{O}_{7}$ layers, which alternate along the c-direction by sharing their $\mathrm{O}$ corners [46]. Each $\mathrm{LaNb}_{2} \mathrm{O}_{7}$ layer represents two consecutive layers (Figure 16a) of $\mathrm{LaNbO}_{3}$ perovskite. The building blocks of the $\mathrm{CuClO}_{2}$ layer are the $\mathrm{CuCl}_{4} \mathrm{O}_{2}$ octahedra with their $\mathrm{O}$ atoms at the apical positions forming a linear $\mathrm{O}-\mathrm{Cu}-\mathrm{O}$ bond aligned along the c-direction. Suppose that the $\mathrm{CuCl}_{4}$ equatorial planes are square in shape with four-fold rotational symmetry around the $\mathrm{O}-\mathrm{Cu}-\mathrm{O}$ axis (Figure $16 \mathrm{~b}, \mathrm{c}$ ), and such $\mathrm{CuCl}_{4} \mathrm{O}_{2}$ octahedra corner-share their $\mathrm{Cl}$ atoms to form a perovskite layer $\mathrm{CuClO}_{2}$. Then, the highest-occupied d-states of each $\mathrm{CuCl}_{4} \mathrm{O}_{2}$ octahedron are degenerate and have three electrons to accommodate, causing a Jahn-Teller instability of each $\mathrm{CuCl}_{4} \mathrm{O}_{2}$ octahedron. In the $\mathrm{CuClO}_{2}$ layer, the $\mathrm{CuCl}_{4} \mathrm{O}_{2}$ octahedra must undergo a cooperative Jahn-Teller distortion. In the tetragonal structure (SG, $\mathrm{P} 4 / \mathrm{mmm})$ of $(\mathrm{CuCl}) \mathrm{LaNb}_{2} \mathrm{O}_{7}$ [46], each $\mathrm{Cl}$ site is split into four positions (Figure 16d). A Jahn-Teller distortion available to such a $\mathrm{CuCl}_{4} \mathrm{O}_{2}$ octahedron is an axial-elongation, in which one linear $\mathrm{Cl}-\mathrm{Cu}-\mathrm{Cl}$ bond is shortened while lengthening the other linear $\mathrm{Cl}-\mathrm{Cu}-\mathrm{Cl}$ bond, as shown in Figure 16e, which can be simplified as in Figure 16f. By choosing one of the four split positions from each $\mathrm{Cl}$ site, it is possible to construct the $\mathrm{CuClO}_{2}$ layer with a cooperative Jahn-Teller distortion, as presented in Figure $17 \mathrm{a}$, which shows the $\mathrm{Cu}-\mathrm{Cl}-\mathrm{Cu}-\mathrm{Cl}$ zigzag chains running along the b-direction with the plane of each $\mathrm{CuCl}_{2} \mathrm{O}_{2}$ rhombus perpendicular to the $\mathrm{a}-\mathrm{b}$ plane. Each $\mathrm{Cl}$ site has four split positions when all these four possibilities occur equally.

With the local coordinate axes of each $\mathrm{CuCl}_{2} \mathrm{O}_{2}$ rhombus taken as in Figure 17a, then the magnetic orbital of the $\mathrm{Cu}^{2+}$ ion can be described as the $\mathrm{x}^{2}-\mathrm{z}^{2}$ state in which $\mathrm{Cu} \mathrm{x} \mathrm{x}^{2}-\mathrm{z}^{2}$ orbital makes $\sigma$-antibonding interactions with the p-orbitals of $\mathrm{O}$ and $\mathrm{Cl}$. Extended Hückel tight binding calculations [47] for the $\mathrm{CuCl}_{2} \mathrm{O}_{2}$ rhombus show that in the magnetic orbital 
of the $\mathrm{CuCl}_{2} \mathrm{O}_{2}$ rhombus, the $\mathrm{Cu}^{2+}$ ion is described by $0.646\left(3 z^{2}-\mathrm{r}^{2}\right)-0.272\left(\mathrm{x}^{2}-\mathrm{y}^{2}\right)$. The latter is rewritten as:

$$
0.646\left(3 z^{2}-r^{2}\right)-0.272\left(x^{2}-y^{2}\right)
$$$$
\approx\left[2\left(3 z^{2}-r^{2}\right)-\left(x^{2}-y^{2}\right)\right] / 3
$$

$\propto\left(\mathrm{z}^{2}-\mathrm{x}^{2}\right)+0.25\left(\mathrm{x}^{2}-\mathrm{y}^{2}\right) \approx\left(\mathrm{z}^{2}-\mathrm{x}^{2}\right)$

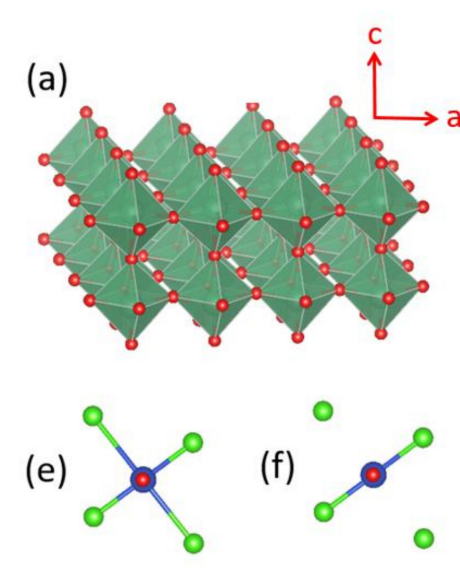

(b)

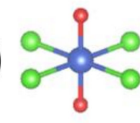

(c)<smiles></smiles>

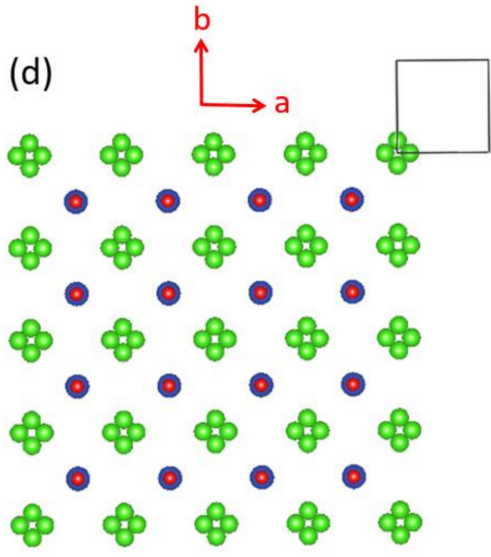

Figure 16. (a) One $\mathrm{LaNb}_{2} \mathrm{O}_{7}$ layer of tetragonal $(\mathrm{CuCl}) \mathrm{LaNb}_{2} \mathrm{O}_{7}$, where the La atoms at the 12 coordinate sites formed by eight corner-sharing $\mathrm{NbO}_{6}$ octahedra are not shown for simplicity. (b) Perspective and (c) projection views of a CuCl $\mathrm{O}_{2}$ octahedron with 4-fold rotational symmetry around the $\mathrm{O}-\mathrm{Cu}-\mathrm{O}$ axis aligned along the c-direction. (d) A projection view of a $\mathrm{CuClO}_{2}$ layer of tetragonal $(\mathrm{CuCl}) \mathrm{LaNb}_{2} \mathrm{O}_{7}$ in which every $\mathrm{Cl}$ site is split into four positions. (e,f) Projection views of the Jahn-Teller distorted $\mathrm{CuCl}_{4} \mathrm{O}_{2}$ octahedron, where the axially elongated $\mathrm{Cu}-\mathrm{Cl}$ bonds are shown in (e), but are not shown in (f) for simplicity.
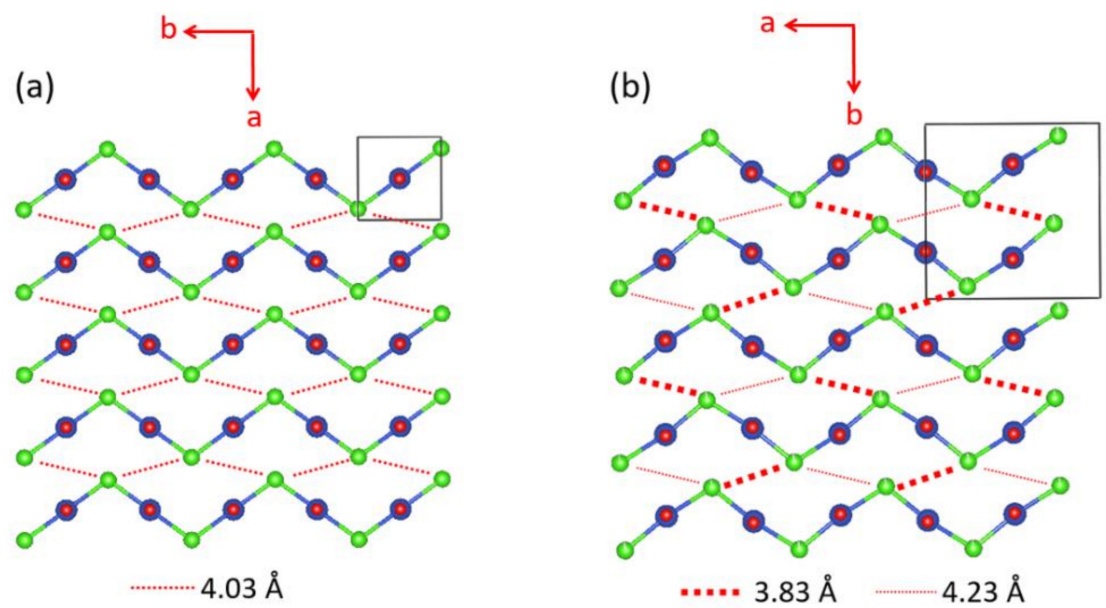

Figure 17. The structures of the $\mathrm{CuClO}_{2}$ layer with cooperative Jahn-Teller distortion in (a) the tetragonal and $(\mathbf{b})$ orthorhombic phases of $(\mathrm{CuCl}) \mathrm{LaNb}_{2} \mathrm{O}_{7}$. The $\mathrm{Cl}-\mathrm{Cu}-\mathrm{Cl}$ unit is linear in the tetragonal structure, but is slightly bent in the orthorhombic structure. The latter has an important consequence on the spin exchanges.

Namely, it is dominated by the $\left(\mathrm{z}^{2}-\mathrm{x}^{2}\right)$ character. This is consistent with the NMR/NQR study of $(\mathrm{CuCl}) \mathrm{LaNb}_{2} \mathrm{O}_{7}$, which showed that the d-state of the $\mathrm{Cu}^{2+}$ ion is mostly characterized by $3 z^{2}-r^{2}$ with some contribution of $x^{2}-y^{2}$ [48].

We note that the $\mathrm{CuCl}_{2} \mathrm{O}_{2}$ rhombuses of each $\mathrm{Cu}-\mathrm{Cl}-\mathrm{Cu}-\mathrm{Cl}$ zigzag chain make $\mathrm{Cu}-\mathrm{Cl} \ldots \mathrm{Cl}-\mathrm{Cu}$ contacts of $\mathrm{Cl} \ldots \mathrm{Cl}=4.03 \AA$ with its adjacent zigzag chains (Figure 17a). The $\mathrm{Cl}$ p-orbitals of the $\left(\mathrm{z}^{2}-\mathrm{x}^{2}\right)$ magnetic orbital (Figure 18a) are pointed approximately 
along the $\mathrm{Cl} \ldots \mathrm{Cl}$ contact, so their overlap is substantial. In all other spin exchange paths, the $\mathrm{Cl}$ p-orbitals in their magnetic orbitals are not arranged to overlap well. Thus, only the exchange along the $\mathrm{Cu}-\mathrm{Cl} \ldots \mathrm{Cl}-\mathrm{Cu}$ direction is expected to be substantially AFM. Then the spin lattice of the $\mathrm{CuClO}_{2}$ layer would be 1D Heisenberg uniform AFM chains running along the $\mathrm{Cu}-\mathrm{Cl} \ldots \mathrm{Cl}-\mathrm{Cu}$ directions, e.g., the $(\mathrm{a}+2 \mathrm{~b})$ - and $(\mathrm{a}-2 \mathrm{~b})$-directions (Figure 17a). Uniform AFM chains do not have a spin gap, but the magnetic properties of $(\mathrm{CuCl}) \mathrm{LaNb}_{2} \mathrm{O}_{7}$ reveal a spin gap behavior [49]. Thus, the tetragonal structure of $(\mathrm{CuCl}) \mathrm{LaNb}_{2} \mathrm{O}_{7}$ is inconsistent with experiment. If one discards the possibility of cooperative Jahn-Teller distortions, one can generate several nonuniform clusters made up of distorted $\mathrm{CuCl}_{4} \mathrm{O}_{2}$ octahedra [50]. However, the latter would lead to several different spin gaps rather than that observed by Kageyama et al. [49], who showed that the magnetic susceptibility of $(\mathrm{CuCl}) \mathrm{LaNb}_{2} \mathrm{O}_{7}$ can be approximated by an isolated spin dimer model with the intradimer distance of approximately $8.8 \AA$, which corresponds to the fourth-nearest-neighbor $\mathrm{Cu}$... $\mathrm{Cu}$ distance. The spin-gap behavior of $(\mathrm{CuCl}) \mathrm{LaNb}_{2} \mathrm{O}_{7}$ was surprising, given the belief of the square lattice arrangement of $\mathrm{Cu}^{2+}$ ions is spin-frustrated $[49,51,52]$. This led to several DFT studies designed to find the precise crystal structure of $(\mathrm{CuCl}) \mathrm{LaNb}_{2} \mathrm{O}_{7}$ [53-55], leading to the conclusion that an orthorhombic structure of space group Pbam is correct for $(\mathrm{CuCl}) \mathrm{LaNb}_{2} \mathrm{O}_{7}[54,55]$.

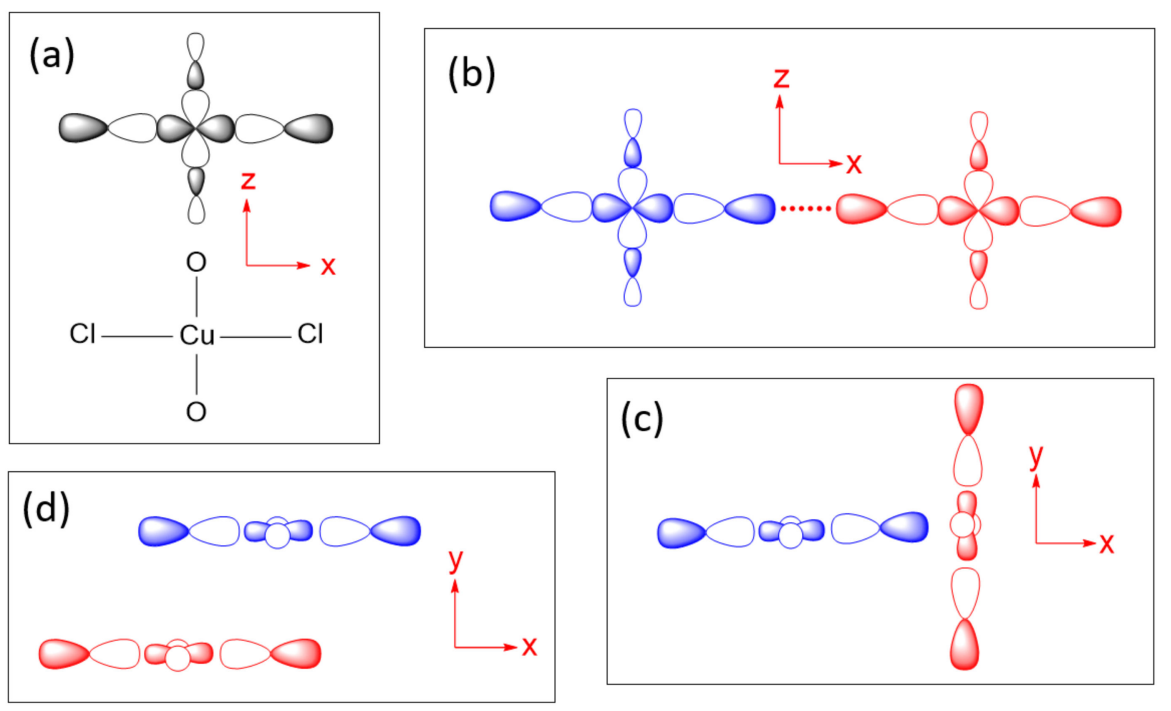

Figure 18. (a) The magnetic orbital lying in the $\mathrm{CuCl}_{2} \mathrm{O}_{2}$ rhombus plane, which is best described as the $x^{2}-z^{2}$ state. $(\mathbf{b}-\mathbf{d})$ Three different types of the magnetic orbital arrangements found in the $\mathrm{CuClO}_{2}$ layers of orthorhombic $\mathrm{CuCl}\left(\mathrm{LaV}_{2} \mathrm{O}_{7}\right)$. In this idealized representation of the $\mathrm{CuCl}_{2} \mathrm{O}_{2}$ rhombus, the slight bending of the $\mathrm{Cl}-\mathrm{Cu}-\mathrm{Cl}$ linkage is neglected for clarity.

The cause for the spin gap behavior of $(\mathrm{CuCl}) \mathrm{LaNb}_{2} \mathrm{O}_{7}$ is found from its orthorhombic structure (SG, Pbam) (Figure 17b) [56,57], in which the arrangement of the $\mathrm{Cu}^{2+}$ ions are no longer tetragonal so that adjacent $\mathrm{Cu}-\mathrm{Cl}-\mathrm{Cu}-\mathrm{Cl}$ zigzag chains have two kinds of $\mathrm{Cl}$ ... Cl contacts (i.e., 3.83 and $4.23 \AA$ ), and the $\mathrm{Cu}-\mathrm{Cl}$.. Cl-Cu chains become alternating with shorter and longer $\mathrm{Cl} \ldots \mathrm{Cl}$ contacts. Furthermore, although the $\mathrm{Cl}-\mathrm{Cu}-\mathrm{Cl}$ unit of each $\mathrm{CuCl}_{2} \mathrm{O}_{2}$ rhombus is slightly bent in the orthorhombic structure, the latter has an important consequence on the spin exchanges (see below).

The six spin exchange paths $\mathrm{J}_{1}-\mathrm{J}_{6}$ of the $\mathrm{CuClO}_{2}$ layer are depicted in Figure 19a. In the spin exchanges $\mathrm{J}_{1}$ and $\mathrm{J}_{2}$, the $\mathrm{Cl}$ p-orbital tails are approximately pointed toward each other (Figure 18b). $\mathrm{J}_{3}$ is a $\mathrm{Cu}-\mathrm{Cl}-\mathrm{Cu}$ exchange with $\angle \mathrm{Cu}-\mathrm{Cl}-\mathrm{Cu}$ angle somewhat greater than $90^{\circ}$ (namely, $109.0^{\circ}$ ). In $\mathrm{J}_{4}$ and $\mathrm{J}_{5}$, the $\mathrm{Cl}$ p-orbital tails are approximately orthogonal to each other (Figure 18c), but they differ due to the bending in the $\mathrm{Cl}-\mathrm{Cu}-\mathrm{Cl}$ units. In the $\mathrm{J}_{6}$ path, the $\mathrm{Cl}$ p-orbital tails are not pointed toward to each other but are approximately 
parallel to each other (Figure 18d). In the exchange paths $\mathrm{J}_{1}$ and $\mathrm{J}_{2}$, the $\mathrm{Cu}-\mathrm{Cl} \ldots \mathrm{Cl}$ linkage is more linear and the $\mathrm{Cl} \ldots \mathrm{Cl}$ contact is shorter in $\mathrm{J}_{1}$. This suggests that $\mathrm{J}_{1}$ is more strongly AFM than $\mathrm{J}_{2}$, thus the spin lattice of the $\mathrm{CuClO}_{2}$ layer is an alternating AFM chain. In agreement with this argument, the energy-mapping analysis based on DFT+U calculations shows that $\mathrm{J}_{1}=87.5 \mathrm{~K}$ and $\mathrm{J}_{2} / \mathrm{J}_{1}=0.18$. In addition, this analysis reveals that $\mathrm{J}_{3}-\mathrm{J}_{6}$ are all FM with $\mathrm{J}_{3} / \mathrm{J}_{1}=-0.39, \mathrm{~J}_{4} / \mathrm{J}_{1}=-0.38, \mathrm{~J}_{5} / \mathrm{J}_{1}=-0.14$ and $\mathrm{J}_{5} / \mathrm{J}_{1}=-0.04$ [56]. It is of interest to note that the strongest AFM exchange $\mathrm{J}_{1}$ is the fourth-nearest-neighbor spin exchange, with a $\mathrm{Cu}$... Cu distance of $8.53 \AA$ [49]. As shown in Figure 19b, the spins of the $\mathrm{CuClO}_{2}$ layer form alternating AFM chains. Chemically, the $\mathrm{Cu}-\mathrm{Cl}$ zigzag chains run along the a-direction. In terms of magnetic bonding, however, the spins of the $\mathrm{CuClO}_{2}$ layer consist of $\mathrm{J}_{1}-\mathrm{J}_{2}$ alternating AFM chains not only along the $(\mathrm{a}+2 \mathrm{~b})$-direction but also along the ( $-\mathrm{a}$ $+2 b)$-direction. This explains why the magnetic susceptibility of $(\mathrm{CuCl}) \mathrm{LaNb}_{2} \mathrm{O}_{7}$ exhibits a spin gap behavior. Due to the bending of the $\mathrm{Cl}-\mathrm{Cu}-\mathrm{Cl}$ units, the $\mathrm{Cl}$ p-orbital tail of one $\mathrm{CuCl}_{2} \mathrm{O}_{2}$ rhombus is pointed toward one $\mathrm{Cl}$ atom (away from both $\mathrm{Cl}$ atoms) of the other rhombus in the $\mathrm{J}_{4}\left(\mathrm{~J}_{5}\right)$ path. This makes $\mathrm{J}_{4}$ more strongly FM than $\mathrm{J}_{5}$ is. $\mathrm{J}_{3}$ is strongly FM despite the fact that the $\angle \mathrm{Cu}-\mathrm{Cl}-\mathrm{Cu}$ angle is somewhat greater than $90^{\circ}$, probably because the $\mathrm{Cl} 3 \mathrm{p}$ orbital tails are more diffuse than the $2 \mathrm{p}$-orbital tails of the second-row ligand (e.g., O). If the spin lattice of the $\mathrm{CuClO}_{2}$ layer is described by using the three strongest spin exchanges, namely, the AFM exchange $\mathrm{J}_{1}$ as well as the FM exchanges $\mathrm{J}_{3}$ and $\mathrm{J}_{4}$, then the resulting spin lattice is topologically equivalent to the Shastry-Sutherland spin lattice (Figure 19c) [56,58].

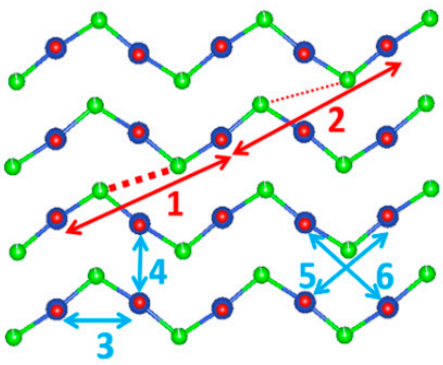

(a)

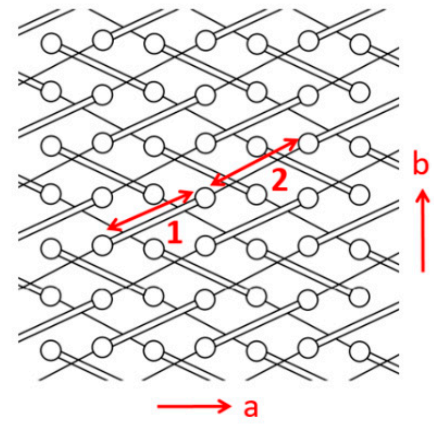

(b)

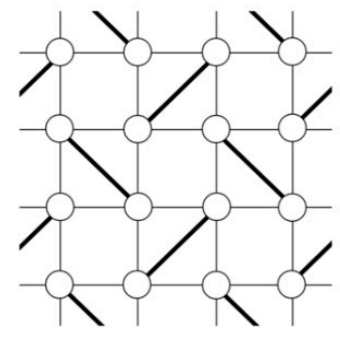

(c)

Figure 19. (a) Six spin exchange paths of the $\mathrm{CuClO}_{2}$ layer in orthorhombic $\mathrm{CuCl}\left(\mathrm{LaV}_{2} \mathrm{O}_{7}\right)$, and the numbers 1-6 refer to $\mathrm{J}_{1}-\mathrm{J}_{6}$, respectively. (b) The $\mathrm{J}_{1}-\mathrm{J}_{2}$ AFM alternating chains the $\mathrm{CuClO}_{2}$ layer. Only the $\mathrm{Cu}^{2+}$ ions are shown as empty circles, with $\mathrm{J}_{1}$ and $\mathrm{J}_{2}$ paths represented by cylinders and lines, respectively. (c) The simplified spin lattice of the $\mathrm{CuClO}_{2}$ layer generated by the three strongest spin exchanges, which is the Shastry-Sutherland spin lattice.

\subsection{Two Dimensional Magnetic Character of Azurite $\mathrm{Cu}_{3}\left(\mathrm{CO}_{3}\right)_{2}(\mathrm{OH})_{2}$}

Early interests in the magnetic properties of the mineral Azurite, $\mathrm{Cu}_{3}\left(\mathrm{CO}_{3}\right)_{2}(\mathrm{OH})_{2}$, in which $\mathrm{Cu}^{2+}$ ions are coordinated with molecular anions $\mathrm{CO}_{3}{ }^{2-}$ and $\mathrm{OH}^{-}$, focused mainly on the paramagnetic AFM ordering transition of the $\mathrm{Cu}^{2+}$ moments that occurs at about $1.86 \mathrm{~K}$ [59-63]. Renewed interests in the properties of $\mathrm{Cu}_{3}\left(\mathrm{CO}_{3}\right)_{2}(\mathrm{OH})_{2}$ arose from low-temperature high-field magnetization measurements by Kikuchi et al. [64], who detected a magnetization plateau extending over a wide field interval between 16 and $26 \mathrm{~T}$ or 11 and $30 \mathrm{~T}$, depending on the crystal orientation. Only one-third of the Cu magnetic moments saturate in these field ranges whereas complete saturation of all $\mathrm{Cu}$ moments occurs above $32.5 \mathrm{~T}$ [64]. In $\mathrm{Cu}_{3}\left(\mathrm{CO}_{3}\right)_{2}(\mathrm{OH})_{2}$, the $\mathrm{Cu}^{2+}$ ions form $\mathrm{CuO}_{4}$ square planar units with the $\mathrm{CO}_{3}{ }^{2-}$ and $\mathrm{OH}^{-}$ions. In each $\mathrm{CuO}_{4}$ unit, two $\mathrm{O}$ atoms come from two $\mathrm{CO}_{3}{ }^{2-}$ ions, and the remaining two $\mathrm{O}$ atoms from two $\mathrm{OH}^{-}$ions. These $\mathrm{CuO}_{4}$ units form $\mathrm{Cu}_{2} \mathrm{O}_{6}$ edge-sharing dimers, which alternate with $\mathrm{CuO}_{4}$ monomers by corner-sharing to make a diamond chain (Figure 20a,b). Guided by the crystal structure, Kikuchi et al. [64] explained 
their results on $\mathrm{Cu}_{3}\left(\mathrm{CO}_{3}\right)_{2}(\mathrm{OH})_{2}$ by considering spin frustration in the diamond chains (Figure 20b), to conclude that all spin exchange constants $\left(\mathrm{J}_{1}, \mathrm{~J}_{2}\right.$ and $\left.\mathrm{J}_{3}\right)$ in the diamond chains are AFM with $\mathrm{J}_{2}$ being the dominant exchange, and that the moment of the $\mathrm{Cu}^{2+}$ ion of the monomer is susceptible to external magnetic fields because its spin exchanges with the two adjacent dimers (i.e., $2 \mathrm{~J}_{1}+2 \mathrm{~J}_{3}$ ) are nearly canceled. In questioning this scenario, $\mathrm{Gu}$ et al. and Rule et al. suggested that one of the monomer-dimer spin exchanges is FM, implying the absence of spin frustration [65-67]. The diamond chain picture had to be revised when the spin exchanges of $\mathrm{Cu}_{3}\left(\mathrm{CO}_{3}\right)_{2}(\mathrm{OH})_{2}$, evaluated using the energy-mapping analysis [68], showed that, although Kikuchi et al.'s description of the diamond chain was correct, $\mathrm{Cu}_{3}\left(\mathrm{CO}_{3}\right)_{2}(\mathrm{OH})_{2}$ is a $2 \mathrm{D}$ spin lattice made up of inter-linked diamond chains.

(a)<smiles>[3H]C[3H]</smiles>

(c)

(d)

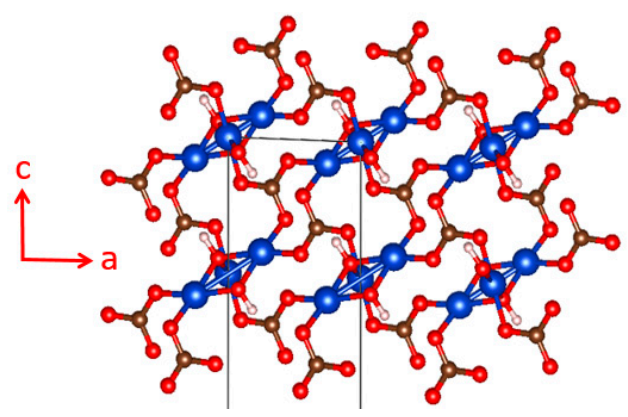

(b)

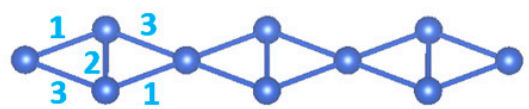

$J_{1}: 3.255 \AA \quad J_{2}: 2.988 \AA \quad J_{3}: 3.289 \AA$

(e)

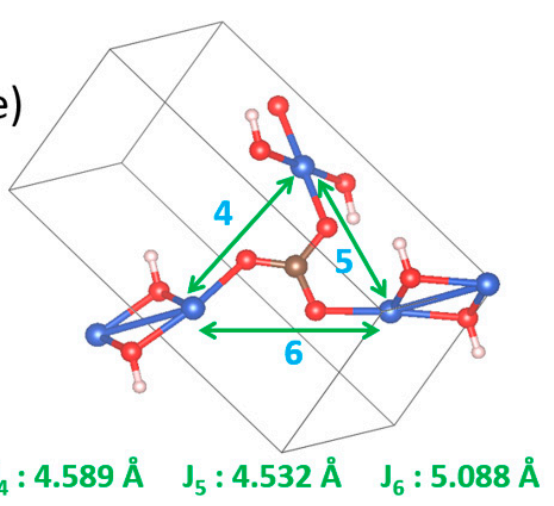

Figure 20. (a) The diamond chain made up of $\mathrm{CuO}_{4}$ square planar units. (b) Definition of the three spin exchanges $\mathrm{J}_{1}-\mathrm{J}_{3}$ in a diamond chain. (c) A projection view of the diamond chain along the chain direction. (d) The structure of Azurite viewed along the diamond chain direction. (e) Definition of the three spin exchanges $\mathrm{J}_{4}-\mathrm{J}_{6}$ between adjacent diamond chains.

In the three-dimensional (3D) structure of $\mathrm{Cu}_{3}\left(\mathrm{CO}_{3}\right)_{2}(\mathrm{OH})_{2}$, the diamond chains are interconnected by the $\mathrm{CO}_{3}{ }^{2-}$ ions. Using the projection view of the diamond chain along the chain direction (Figure 20c), the 3D structure of $\mathrm{Cu}_{3}\left(\mathrm{CO}_{3}\right)_{2}(\mathrm{OH})_{2}$ can be represented as in Figure 20d, which shows that each $\mathrm{CO}_{3}{ }^{2-}$ ion bridges three different diamond chains. The spin exchange paths of interest for $\mathrm{Cu}_{3}\left(\mathrm{CO}_{3}\right)_{2}(\mathrm{OH})_{2}$ are $\mathrm{J}_{1}-\mathrm{J}_{3}$ in each diamond chain (Figure 20b), as well as $\mathrm{J}_{4}-\mathrm{J}_{6}$ between diamond chains (Figure 20e). In the diamond unit of Azurite (Figure 21a), the two bridging $\mathrm{O}$ atoms of the edge-sharing dimer $\mathrm{Cu}_{2} \mathrm{O}_{6}$ form $\mathrm{O}-\mathrm{H}$ bonds. Thus, the spin exchange $\mathrm{J}_{2}$ (Figure 20b) between the two $\mathrm{Cu}^{2+}$ ions in the edgesharing dimer $\mathrm{Cu}_{2} \mathrm{O}_{6}$ are expected to be strongly AFM, as discussed in Section 4.2. The spin exchanges $\mathrm{J}_{1}$ and $\mathrm{J}_{3}$ of the diamond (Figure 20b) would be similar in strength because their two $\mathrm{Cu}-\mathrm{O}-\mathrm{Cu}$ exchange paths are nearly equivalent due to the near perpendicular arrangement the $\mathrm{CuO}_{4}$ monomer plane to the $\mathrm{Cu}_{2} \mathrm{O}_{6}$ dimer plane (Figure 21a). Due to the perpendicular arrangement of the two planes, $\mathrm{J}_{1}$ and $\mathrm{J}_{3}$ are expected to be weakly AFM and smaller than $\mathrm{J}_{2}$. What is difficult to predict without quantitative calculations is the relative strengths of the inter-chain exchanges $\mathrm{J}_{4}-\mathrm{J}_{6}$ (Figure 20e). For example, we consider the $\mathrm{J}_{4}$ exchange path shown in Figure 21b. The $\mathrm{Op}_{\pi}$ and $\mathrm{Op}_{\sigma}$ orbitals of the $\mathrm{CO}_{3}{ }^{2-}$ ion interact with the p-orbital tail of the $\mathrm{Cu}^{2+}$ ion magnetic orbital (Figure 21c), which depend not only on the $\angle \mathrm{C}-\mathrm{O}-\mathrm{Cu}$ bond angle, but also on the dihedral angles associated with the spin exchange path (e.g., $\angle \mathrm{O}-\mathrm{C}-\mathrm{O}-\mathrm{Cu}$ dihedral angle). It is necessary to resort to the energy-mapping analysis based on $\mathrm{DFT}+\mathrm{U}$ calculations to find the relative strengths of the spin exchanges $\mathrm{J}_{1}-\mathrm{J}_{6}$. The arrangement of these spin exchange paths in Azurite is presented in Figure 22a. Results of our analysis for $\mathrm{J}_{1}-\mathrm{J}_{4}$ are summarized in Table 1. 
(a)

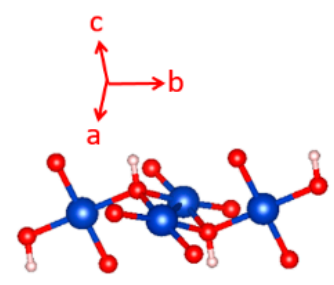

(c)

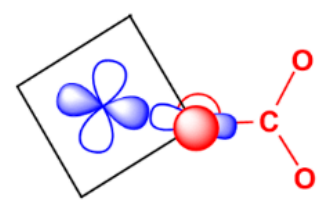

(b)
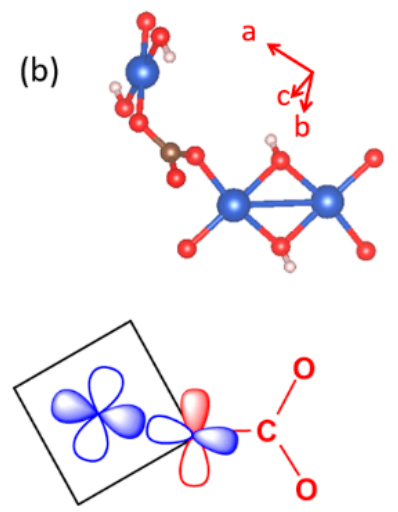

Figure 21. (a) A diamond unit of Azurite. (b) Arrangement of the $\mathrm{CuO}_{4}$ monomer and $\mathrm{Cu}_{2} \mathrm{O}_{6}$ dimer associated with the spin exchange path $\mathrm{J}_{4}$. (c) Interaction of the $\mathrm{O} \mathrm{p}_{\pi}$ and $\mathrm{O} \mathrm{p}_{\sigma}$ orbitals of the $\mathrm{CO}_{3}{ }^{2-}$ ion with the p-orbital tail of the $\mathrm{Cu}^{2+}$ ion magnetic orbital.

(a)

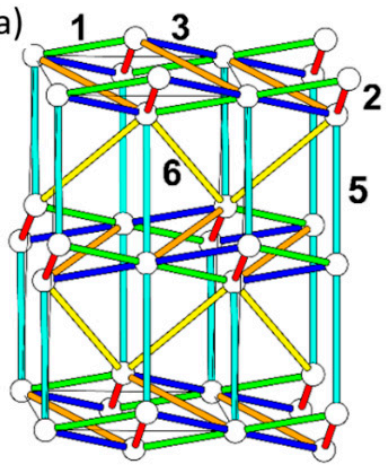

(b)

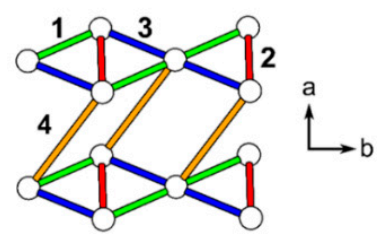

(c)

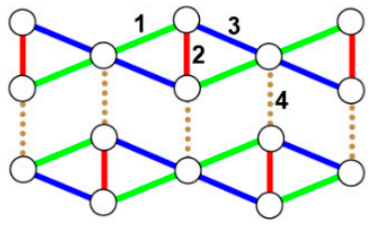

Figure 22. (a) Arrangement of the spin exchange paths $\mathrm{J}_{1}-\mathrm{J}_{6}$ in Azurite. (b) Arrangement of the four strongest spin exchanges $\mathrm{J}_{1}-\mathrm{J}_{4}$ in Azurite. (c) 2D spin lattice describing Azurite.

Table 1. Values of the spin exchanges $\mathrm{J}_{1}-\mathrm{J}_{6}$ determined by DFT+U calculations with $\mathrm{U}_{\text {eff }}=4$ and $5 \mathrm{eV}$.

\begin{tabular}{ccc}
\hline & $\mathrm{U}_{\text {eff }}=\mathbf{4}$ & $\mathrm{U}_{\text {eff }}=\mathbf{5}$ \\
\hline $\mathrm{J}_{2}$ & $241.5 \mathrm{~K}$ & $194.6 \mathrm{~K}$ \\
\hline $\mathrm{J}_{3} / \mathrm{J}_{2}$ & 0.28 & 0.27 \\
\hline $\mathrm{J}_{1} / \mathrm{J}_{2}$ & 0.21 & 0.19 \\
\hline $\mathrm{J}_{4} / \mathrm{J}_{2}$ & 0.17 & 0.16 \\
\hline $\mathrm{J}_{5} / \mathrm{J}_{2}$ & -0.01 & -0.02 \\
\hline $\mathrm{J}_{6} / \mathrm{J}_{2}$ & 0.04 & 0.03 \\
\hline
\end{tabular}

Our energy-mapping analysis shows that $\mathrm{J}_{5}$ and $\mathrm{J}_{6}$ are negligibly weak compared with $\mathrm{J}_{1}-\mathrm{J}_{4}$. The latter four exchanges are all AFM; $\mathrm{J}_{2}$ is the strongest while $\mathrm{J}_{1}, \mathrm{~J}_{3}$ and $\mathrm{J}_{4}$ are comparable in magnitude, which is in support of Kikuchi et al.'s deduction of the spin exchanges $\mathrm{J}_{1}-\mathrm{J}_{3}$ for the diamond chain. The values of $\mathrm{J}_{1}-\mathrm{J}_{4}$ are smaller from the DFT+U calculations with $\mathrm{U}_{\text {eff }}=5 \mathrm{eV}$ than from those with $\mathrm{U}_{\text {eff }}=4 \mathrm{eV}$. This is understandable because the $\mathrm{J}_{\mathrm{AF}}$ component decreases with increasing the on-site repulsion $\mathrm{U}$ (Equation (19)). The strengths of the exchanges $\mathrm{J}_{1}-\mathrm{J}_{4}$ decrease in the order, $\mathrm{J}_{2}>>\mathrm{J}_{3} \approx \mathrm{J}_{1}>\mathrm{J}_{4}$. Thus, in the spin lattice of Azurite, the diamond chains defined by the intrachain exchanges $\mathrm{J}_{2}, \mathrm{~J}_{3}$ and $\mathrm{J}_{1}$ interact by the interchain exchange $\mathrm{J}_{4}$ (Figure 22b). Therefore, the spin lattice of Azurite is a $2 \mathrm{D}$ spin lattice described by the exchanges $\mathrm{J}_{1}-\mathrm{J}_{4}$ depicted in Figure 22c. $\mathrm{J}_{3}$ and $\mathrm{J}_{1}$ are practically equal in strength; therefore, use of a symmetrical diamond chain (i.e., with 
the approximation $\mathrm{J}_{3} \approx \mathrm{J}_{1}$ ) would be a good approximation. In any event, it is crucial not to neglect the interchain exchange $\mathrm{J}_{4}$ because it is comparable in magnitude to $\mathrm{J}_{3}$ and $\mathrm{J}_{1}$. Alternatively, the spin lattice can be described in terms of the alternating AFM chains defined by $\mathrm{J}_{2}$ and $\mathrm{J}_{4}$. The $2 \mathrm{D}$ spin lattice consists of these alternating AFM chains that are spin-frustrated by the interchain exchanges $\mathrm{J}_{1}$ and $\mathrm{J}_{3}$.

The importance of interchain exchange indicating that the correct spin lattice of Azurite is not a diamond chain but a 2D net in which the diamond chains are interconnected by the spin exchange spin $\mathrm{J}_{4}$ was recognized by Kang et al. in 2009 [68]. This finding, though controversial and vigorously disputed in the beginning, is now accepted as a prerequisite for correctly describing Azurite [69]. The presence of an interchain exchange naturally allows one to understand a long-range AFM order and explain gapped modes in the spin dynamics along the diamond chains [70], and the magnetic contribution to the thermal conductivity [71].

\section{Concluding Remarks}

In this review, we discussed the theoretical foundations of the concept of spin exchanges and analyzed which electronic factors affect their signs and strengths. Noting that a spin exchange between two magnetic ions is mediated by the ligand p-orbital tails, we derived several qualitative rules for predicting whether a given $\mathrm{M}-\mathrm{L} \ldots \mathrm{L}-\mathrm{M}$ or $\mathrm{M}-\mathrm{L} \ldots$ A ... L-M exchange would be AFM or FM by inspecting the arrangement of their ligand p-orbital tails in the exchange paths. As long as the L ... L distance is in the vicinity of the van der Waals distance, the M-L ... L-M or M-L ... A ... L-M spin exchange can be strong and often stronger than the $\mathrm{M}-\mathrm{L}-\mathrm{M}$ exchanges. In searching for the spin lattice relevant for a given magnetic solid, therefore, it is crucial not to omit the M-L . . L L-M and $\mathrm{M}-\mathrm{L} \ldots \mathrm{A}$... L-M spin exchanges when present. The qualitative rules on the $\mathrm{M}-\mathrm{L}$ ... L-M and M-L ... A ... L-M exchanges, described in Section 4.3, can be used together with the Goodenough-Kanamori rules on M-L-M spin exchanges in selecting a proper set of spin exchanges to evaluate using the energy-mapping analysis.

The important aspect emerging from our discussions is that the nature of a spin exchange is determined by the interactions between the magnetic orbitals. These are governed by the ligand p-orbitals, not by the metal d-orbitals. The essential role that the metal d-orbitals play in any spin exchange is rather indirect. In a magnetic orbital of an $\mathrm{ML}_{n}$ polyhedron, the metal d-orbital selects with which ligand p-orbitals it combines and hence determines the nature of the p-orbital tails in the magnetic orbital. The spin exchange between magnetic ions, namely, the interaction between their magnetic orbitals, rests upon the interaction between their p-orbital tails.

Author Contributions: Conceptualization, M.-H.W.; methodology, M.-H.W.; validation, M.-H.W., H.-J.K. and R.K.K.; formal analysis, M.-H.W.; investigation, M.-H.W., H.-J.K. and R.K.K.; writingoriginal draft preparation, M.-H.W.; writing-review and editing, M.-H.W., H.-J.K. and R.K.K.; visualiza-tion, M.-H.W.; supervision, M.-H.W.; project administration, M.-H.W.; funding acquisition, H.-J.K. All authors have read and agreed to the published version of the manuscript.

Funding: The work at Kyung Hee University was supported by the Basic Science Research Program through the National Research Foundation of Korea (NRFK) funded by the Ministry of Education (2020R1A6A1A03048004).

Institutional Review Board Statement: Not applicable.

Informed Consent Statement: Not applicable.

Acknowledgments: H.J.K. thanks the NRFK for the fund 2020R1A6A1A03048004.

Conflicts of Interest: The authors declare no conflict of interest. 


\section{References}

1. Whangbo, M.-H.; Koo, H.-J.; Dai, D. Spin exchange interactions and magnetic structures of extended magnetic solids with localized spins: Theoretical descriptions on formal, quantitative and qualitative levels. J. Solid State Chem. 2003, 176, $417-481$. [CrossRef]

2. Xiang, H.J.; Lee, C.; Koo, H.-J.; Gong, X.; Whangbo, M.-H. Magnetic properties and energy-mapping analysis. Dalton Trans. 2013, 42, 823-853. [CrossRef] [PubMed]

3. Whangbo, M.-H.; Xiang, H.J. Magnetic Properties from the Perspectives of Electronic Hamiltonian: Spin Exchange Parameters, Spin Orientation and Spin-Half Misconception. In Handbook in Solid State Chemistry, Volume 5: Theoretical Descriptions; Wiley: New York, NY, USA, 2017; pp. 285-343.

4. Anderson, P.W. Antiferromagnetism-Theory of superexchange. Phys. Rev. 1950, 79, 350-356. [CrossRef]

5. Goodenough, J.B.; Loeb, A.L. Theory of ionic ordering, crystal distortion, and magnetic exchange due to covalent forces in spinels. Phys. Rev. 1955, 98, 391-408. [CrossRef]

6. Goodenough, J.B. Theory of the role of covalence in the perovskite-type manganites [La, M(II)]MnO3. Phys. Rev. 1955, 100, t564-573. [CrossRef]

7. Kanamori, J. Superexchange interaction and symmetry properties of electron orbitals. J. Phys. Chem. Solids 1959, 10, 87-98. [CrossRef]

8. Goodenough, J.B. Magnetism and the Chemical Bond; Interscience, Wiley: New York, NY, USA, 1963.

9. Martin, R.L. Metal-metal interaction in paramagnetic clusters. In New Pathways of Inorganic Chemistry; Ebsworth, E.A.V., Maddock, A.G., Sharp, A.G., Eds.; Cambridge University Press: Cambridge, UK, 1968; Chapter 9.

10. Pauling, L. The Nature of the Chemical Bond and the Structure of Molecules and Solids: The Introduction to Mode, 3rd ed.; Cornell University: Ithaca, NY, USA, 1960.

11. Dudarev, S.L.; Botton, G.A.; Savrasov, S.Y.; Humphreys, C.J.; Sutton, A.P. Electron-energy-loss spectra and the structural stability of nickel oxide: An LSDA+U study. Phys. Rev. B 1998, 57, 1505-1509. [CrossRef]

12. Heyd, J.; Scuseria, G.E.; Ernzerhof, M. Hybrid functionals based on a screened Coulomb potential. J. Chem. Phys. 2003, 118, 8207. [CrossRef]

13. Xiang, H.J.; Kan, E.J.; Wei, S.-H.; Whangbo, M.-H.; Gong, X.G. Predicting the spin-lattice order of frustrated systems from first principles. Phys. Rev. B 2011, 84, 224429. [CrossRef]

14. Li, X.-Y.; Yu, H.Y.; Lou, F.; Feng, J.-S.; Whangbo, M.-H.; Xiang, H.J. Spin Hamiltonians in Magnets: Theories and Computations. Molecules. submitted for Publication.

15. Hay, P.J.; Thibeault, J.C.; Hoffmann, R. Orbital interactions in metal dimer complexes. J. Am. Chem. Soc. 1975, 97, 4884-4899. [CrossRef]

16. Vasil'ev, A.N.; Ponomarenko, L.A.; Smirnov, A.I.; Antipov, E.V.; Velikodny, Y.A.; Isobe, M.; Ueda, Y. Short-range and long-range magnetic ordering in $\alpha-\mathrm{CuV}_{2} \mathrm{O}_{6}$. Phys. Rev. B 1999, 60, 3021-3024. [CrossRef]

17. Kikuchi, J.; Ishiguchi, K.; Motoya, K.; Itoh, M.; Inari, K.; Eguchi, N.; Akimitsu, J. NMR and neutron scattering studies of quasi one-dimensional magnet $\mathrm{CuV}_{2} \mathrm{O}_{6}$. J. Phys. Soc. Jpn. 2000, 69, 2660-2668. [CrossRef]

18. Prokofiev, A.V.; Kremer, R.K.; Assmus, W. Crystal growth and magnetic properties of $\alpha-\mathrm{CuV}_{2} \mathrm{O}_{6}$. J. Cryst. Growth 2001, 231, 498-505. [CrossRef]

19. Golubev, A.M.; Nuss, J.; Kremer, R.K.; Gordon, E.E.; Whangbo, M.-H.; Ritter, C.; Weber, L.; Wessel, S. Two-dimensional magnetism in $\alpha-\mathrm{CuV}_{2} \mathrm{O}_{6}$. Phys. Rev. B 2020, 102, 014436. [CrossRef]

20. Koo, H.-J.; Lee, C.; Whangbo, M.-H.; McIntyre, G.J.; Kremer, R.K. On the nature of the spin frustration in the $\mathrm{CuO}_{2}$ ribbon chains of $\mathrm{LiCuVO}_{4}$ : Crystal structure determination at $1.6 \mathrm{~K}$, magnetic susceptibility analysis and density functional evaluation of the spin exchange constants. Inorg. Chem. 2011, 50, 3582-3588. [CrossRef]

21. Yamaguchi, M.; Furuta, T.; Ishikawa, M. Calorimetric study of several cuprates with restricted dimensionality. J. Phys. Soc. Jpn. 1996, 65, 2998-3006. [CrossRef]

22. Vasil'ev, A.N.; Ponomarenko, L.A.; Manaka, H.; Yamada, I.; Isobe, M.; Ueda, Y. Quasi-one-dimensional antiferromagnetic spinel compound $\mathrm{LiCuVO}_{4}$. Physica B 2000, 284-288, 1619-1620. [CrossRef]

23. Vasil'ev, A.N.; Ponomarenko, L.A.; Manaka, H.; Yamada, I.; Isobe, M.; Ueda, Y. Magnetic and resonant properties of quasi-onedimensional antiferromagnet $\mathrm{LiCuVO}_{4}$. Phys. Rev. B 2001, 64, 024419. [CrossRef]

24. Gibson, B.J.; Kremer, R.K.; Prokofiev, A.V.; Assmus, W.; McIntyre, G.J. Incommensurate antiferromagnetic order in the S=1/2 quantum chain compound $\mathrm{LiCuVO}_{4}$. Physica B 2004, 350, e253-e256. [CrossRef]

25. Dai, D.; Koo, H.-J.; Whangbo, M.-H. Investigation of the incommensurate and commensurate magnetic superstructures of $\mathrm{LiCuVO}_{4}$ and $\mathrm{CuO}$ on the basis of the isotropic spin exchange and classical spin approximations. Inorg. Chem. 2004, 43, 4026-4035. [CrossRef] [PubMed]

26. Enderele, M.; Mukherjee, C.; Fåk, B.; Kremer, R.K.; Broto, J.-M.; Rosner, H.; Drechsler, S.-L.; Richter, J.; Malek, J.; Prokofiev, A.; et al. Quantum helimagnetism of the frustrated spin-1/2 chain $\mathrm{LiCuVO}_{4}$. Europhys. Lett. 2005, 70, 237-243. [CrossRef]

27. Gordon, E.E.; Derakhshan, S.; Thompson, C.M.; Whangbo, M.-H. Spin-density wave as a superposition of two magnetic states of opposite chirality and its implications. Inorg. Chem. 2018, 57, 9782-9785. [CrossRef] [PubMed]

28. Koo, H.-J.; N, R.S.P.; Orlandi, F.; Sundaresan, A.; Whangbo, M.-H. On Ferro and antiferro spin density waves describing the incommensurate magnetic structure of $\mathrm{NaYNiWO}_{6}$. Inorg. Chem. 2020, 59, 17856-17859. [CrossRef] 
29. Naito, Y.; Sato, K.; Yasui, Y.; Kobayashi, Y.; Kobayashi, Y.; Sato, M. Relationship between magnetic structure and ferroelectricity of $\mathrm{LiVCuO}_{4}$. J. Phys. Soc. Jpn. 2007, 76, 023708. [CrossRef]

30. Xiang, H.J.; Whangbo, M.-H. Density-functional characterization of the multiferroicity in spin spiral chain cuprates. Phys. Rev. Lett. 2007, 99, 257203. [CrossRef] [PubMed]

31. Yasui, Y.; Naito, Y.; Sato, K.; Moyoshi, T.; Sato, M.; Kakurai, K. Studies of Multiferroic System $\mathrm{LiCu}_{2} \mathrm{O}_{2}$ : I. Sample characterization and relationship between magnetic properties and multiferroic nature. J. Phys. Soc. Jpn. 2008, 77, 023712. [CrossRef]

32. Schrettle, F.; Krohns, S.; Lunkenheimer, P.; Hemberger, J.; Büttgen, N.; von Nidda, H.-A.K.; Prokofiev, A.V.; Loidl, A. Switching the ferroelectric polarization in the $\mathrm{S}=1 / 2$ chain cuprate $\mathrm{LiCuVO}_{4}$ by external magnetic fields. Phys. Rev. B 2008, 77, 144101. [CrossRef]

33. Ruff, A.; Krohns, S.; Lunkenheimer, P.; Prokofiev, A.; Loidl, A. Dielectric properties and electrical switching behaviour of the spin-driven multiferroic $\mathrm{LiCuVO}_{4}$. J. Phys. Condens. Matter 2014, 26, 485901. [CrossRef]

34. Ruff, A.; Lunkenheimer, P.; von Nidda, H.-A.K.; Widmann, S.; Prokofiev, A.; Svistov, L.; Loidl, A.; Krohns, S. Chirality-driven ferroelectricity in $\mathrm{LiCuVO}_{4}$. NPJ Quantum Mater. 2019, 4, 24. [CrossRef]

35. Mourigal, M.; Enderle, M.; Kremer, R.K.; Law, J.M.; Fåk, B. Ferroelectricity from spin supercurrents in LiCuVO 4 . Phys. Rev. B 2014, 83, 100409(R). [CrossRef]

36. Banks, M.G.; Heidrich-Meisner, F.; Honecker, A.; Rakoto, H.; Broto, J.-M.; Kremer, R.K. High field magnetization of the frustrated one-dimensional quantum antiferromagnet $\mathrm{LiCuVO}_{4}$. J. Phys. Condens. Matter 2007, 19, 145227. [CrossRef]

37. Hikihara, T.; Kecke, L.; Momoi, T.; Furusaki, A. Vector chiral and multipolar orders in the spin-1/2 frustrated ferromagnetic chain in magnetic field. Phys. Rev. B 2008, 78, 144404. [CrossRef]

38. Mourigal, M.; Enderle, M.; Fåk, B.; Kremer, R.K.; Law, J.M.; Schneidewind, A.; Hiess, A.; Prokofiev, A. Evidence of a bond-nematic phase in $\mathrm{LiCuVO}_{4}$. Phys. Rev. Lett. 2012, 109, 027203. [CrossRef] [PubMed]

39. Orlova, A.; Green, E.L.; Law, J.M.; Gorbunov, D.I.; Chanda, G.; Krämer, S.; Horvatić, M.; Kremer, R.K.; Wosnitza, J.; Rikken, G.L.J.A. Nuclear magnetic resonance signature of the spin-nematic phase in $\mathrm{LiCuVO}_{4}$ at high magnetic fields. Phys. Rev. Lett. 2017, 118, 247201. [CrossRef]

40. Zhitomirsky, M.E.; Tsunetsugu, H. Magnon pairing in quantum spin nematic. Europhys. Lett. 2010, 92, 37001. [CrossRef]

41. Svistova, L.E.; Fujita, T.; Yamaguchi, H.; Kimura, S.; Omura, K.; Prokofiev, A.; Smirnova, A.I.; Honda, Z.; Hagiwara, M. New high magnetic field phase of the frustrated $\mathrm{S}=1 / 2$ Chain compound $\mathrm{LiCuVO}_{4}$. JETP Lett. 2011, 93, 21. [CrossRef]

42. Ueda, H.T.; Momoi, T. Nematic phase and phase separation near saturation field in frustrated ferromagnets. Phys. Rev. B 2013, 87, 144417. [CrossRef]

43. Sato, M.; Hikihara, T.; Momoi, T. Spin-nematic and spin-density-wave orders in spatially anisotropic frustrated magnets in a magnetic field. Phys. Rev. Lett. 2013, 110, 077206. [CrossRef]

44. Starykh, O.A.; Balents, L. Excitations and quasi-one-dimensionality in field-induced nematic and spin density wave states. Phys. Rev. B 2014, 89, 104407. [CrossRef]

45. Büttgen, N.; Nawa, K.; Fujita, T.; Hagiwara, M.; Kuhns, P.; Prokofiev, A.; Reyes, A.P.; Svistov, L.E.; Yoshimura, K.; Takigawa, M. Search for a spin-nematic phase in the quasi-one-dimensional frustrated magnet $\mathrm{LiCuVO}_{4}$. Phys. Rev. B 2014, $90,134401$. [CrossRef]

46. Caruntu, G.; Kodenkandath, T.A.; Wiley, J.B. Neutron diffraction study of the oxychloride layered perovskite, $(\mathrm{CuCl}) \mathrm{LaNb}_{2} \mathrm{O}_{7}$. Mater. Res. Bull. 2002, 37, 593-598. [CrossRef]

47. Hoffmann, R. An extended Hückel theory. I. Hydrocarbons. J. Chem. Phys. 1963, 39, 1397. [CrossRef]

48. Yoshida, M.; Ogata, N.; Takigawa, M.; Yamamura, J.-I.; Ichihara, M.; Kitano, T.; Kageyama, H.; Ajiro, Y.; Yoshimura, K. Magnetic and structural studies of the quasi-two-dimensional spin-gap system $(\mathrm{CuCl}) \mathrm{LaNb}_{2} \mathrm{O}_{7}$. J. Phys. Soc. Jpn. 2007, $76,104703$. [CrossRef]

49. Kageyama, H.; Kitano, T.; Oba, N.; Nishi, M.; Nagai, S.; Hirota, K.; Viciu, L.; Wiley, J.B.; Yasuda, J.; Baba, Y.; et al. Spin-singlet ground state in two-dimensional $\mathrm{S}=1 / 2$ frustrated square lattice: $(\mathrm{CuCl}) \mathrm{LaNb}_{2} \mathrm{O}_{7}$. J. Phys. Soc. Jpn. 2005, 74, 1702-1705. [CrossRef]

50. Whangbo, M.-H.; Dai, D. On the Disorder of the $\mathrm{Cl}$ atom position in and its probable effect on the magnetic properties of (CuCl)LaNb ${ }_{2} \mathrm{O}_{7}$. Inorg. Chem. 2006, 45, 6227-6234. [CrossRef]

51. Kageyama, H.; Yasuda, J.; Kitano, T.; Totsuka, K.; Narumi, Y.; Hagiwara, M.; Kindo, K.; Baba, Y.; Oba, N.; Ajiro, Y.; et al. Anomalous magnetization of two-dimensional $\mathrm{S}=1 / 2$ frustrated square-lattice antiferromagnet $(\mathrm{CuCl}) \mathrm{LaNb}_{2} \mathrm{O}_{7} . J$. Phys. Soc. Jpn. 2005, 74, 3155-3158. [CrossRef]

52. Hiroi, A.K.Z.; Tsujimoto, Y.; Kitano, T.; Kageyama, H.; Ajiro, Y.; Yoshimura, K. Bose-Einstein condensation of quasi-twodimensional frustrated quantum magnet $(\mathrm{CuCl}) \mathrm{LaNb}_{2} \mathrm{O}_{7}$. J. Phys. Soc. Jpn. 2007, 76, 093706.

53. Tsirlin, A.A.; Rosner, H. Structural distortion and frustrated magnetic interactions in the layered copper oxychloride (CuCl)LaNb ${ }_{2} \mathrm{O}_{7}$. Phys. Rev. B 2009, 79, 214416. [CrossRef]

54. Ren, C.-Y.; Cheng, C. Atomic and magnetic structures of $(\mathrm{CuCl}) \mathrm{LaNb}_{2} \mathrm{O}_{7}$ and $(\mathrm{CuBr}) \mathrm{LaNb}_{2} \mathrm{O}_{7}$ : Density functional calculations. Phys. Rev. B 2010, 82, 024404. [CrossRef]

55. Tsirlin, A.A.; Abakumov, A.M.; van Tendeloo, G.; Rosner, H. Interplay of atomic displacements in the quantum magnet, (CuCl)LaNb $2 \mathrm{O}_{7}$. Phys. Rev. B 2010, 82, 054107. [CrossRef] 
56. Tassel, C.; Kang, J.; Lee, C.; Hernandez, O.; Qiu, Y.; Paulus, W.; Collet, E.; Lake, B.; Guidi, T.; Whangbo, M.-H.; et al. Ferromagnetically coupled Shastry-Sutherland quantum spin singlets in $(\mathrm{CuCl}) \mathrm{LaNb}_{2} \mathrm{O}_{7}$. Phys. Rev. Lett. 2010, 105, 167205. [CrossRef] [PubMed]

57. Hernandez, O.J.; Tassel, C.; Nakano, K.; Paulus, W.; Ritter, C.; Collet, E.; Kitada, A.; Yoshimura, K.; Kageyama, H. First singlecrystal synthesis and low-temperature structural determination of the quasi-2D quantum spin compound $(\mathrm{CuCl}) \mathrm{LaNb}_{2} \mathrm{O}_{7}$. Dalton Trans. 2011, 40, 4605-4613. [CrossRef] [PubMed]

58. Furukawa, S.; Dodds, T.; Kim, Y.B. Ferromagnetically coupled dimers on the distorted Shastry-Sutherland lattice: Application to (CuCl)LaNb $\mathrm{O}_{7}$. Phys. Rev. B 2011, 84, 054432. [CrossRef]

59. Spence, R.D.; Ewing, R.D. Evidence for antiferromagnetism in $\mathrm{Cu}_{3}\left(\mathrm{CO}_{3}\right)_{2}(\mathrm{OH})_{2}$. Phys. Rev. 1958, 112, 1544-1545. [CrossRef]

60. Forstat, H.; Taylor, G.; King, B.R. Low-temperature heat capacity of Azurite. J. Chem. Phys. 1959, 31, 929-931. [CrossRef]

61. van der Lugt, W.; Poulis, N.J. Proton magnetic resonance in Azurite. Physica 1959, 25, 1313-1320. [CrossRef]

62. Garber, M.; Wagner, R. The susceptibility of Azurite. Physica 1960, 26, 777. [CrossRef]

63. Love, N.D.; Duncan, T.K.; Bailey, P.T.; Forstat, H. Spin flopping in Azurite. Phys. Lett. A 1970, 33, 290-291. [CrossRef]

64. Kikuchi, H.; Fujii, Y.; Mitsudo, S.; Idehara, T. Magnetic properties of the frustrated diamond chain $\mathrm{compound} \mathrm{Cu}_{3}\left(\mathrm{CO}_{3}\right)_{2}(\mathrm{OH})_{2}$ Phys. B 2003, 329, 967-968. [CrossRef]

65. Gu, B.; Su, G. Comment on "Experimental Observation of the 1/3 Magnetization Plateau in the Diamond-Chain Compound $\mathrm{Cu}_{3}\left(\mathrm{CO}_{3}\right)_{2}(\mathrm{OH})_{2}$. Phys. Rev. Lett. 2006, 97, 089701. [CrossRef] [PubMed]

66. Gu, B.; Su, G. Magnetism and thermodynamics of spin-1/2 Heisenberg diamond chains in a magnetic field. Phys. Rev. B 2007, 75, 174437. [CrossRef]

67. Rule, K.C.; Wolter, A.U.B.; Süllow, S.; Tennant, D.A.; Brühl, A.; Köhler, S.; Wolf, B.; Lang, M.; Schreuer, J. Nature of the spin dynamics and 1/3 magnetization plateau in Azurite. Phys. Rev. Lett. 2008, 100, 117202. [CrossRef] [PubMed]

68. Kang, J.; Lee, C.; Kremer, R.K.; Whangbo, M.-H. Consequences of the intrachain dimer-monomer spin frustration and the interchain dimer-monomer spin exchange in the diamond-chain compound azurite $\mathrm{Cu}_{3}\left(\mathrm{CO}_{3}\right)_{2}(\mathrm{OH})_{2}$. J. Phys. Condens. Matter 2009, 21, 392201. [CrossRef] [PubMed]

69. Jeschke, H.; Opahle, I.; Kandpal, H.; Valentí, R.; Das, H.; Saha-Dasgupta, T.; Janson, O.; Rosner, H.; Brühl, A.; Wolf, B.; et al. Multistep approach to microscopic models for frustrated quantum magnets: The case of the natural mineral Azurite. Phys. Rev. Lett. 2011, 106, 217201. [CrossRef]

70. Rule, K.C.; Tennant, D.A.; Caux, J.-S.; Gibson, M.C.R.; Telling, M.T.F.; Gerischer, S.; Süllow, S.; Lang, M. Dynamics of azurite $\mathrm{Cu}_{3}\left(\mathrm{CO}_{3}\right)_{2}(\mathrm{OH})_{2}$ in a magnetic field as determined by neutron scattering. Phys. Rev. B 2011, 84, 184419. [CrossRef]

71. Wu, C.; Song, J.D.; Zhao, Z.Y.; Shi, J.; Xu, H.S.; Zhao, J.Y.; Liu, X.G.; Zhao, X.; Sun, X.F. Thermal conductivity of the diamond-chain compound $\mathrm{Cu}_{3}\left(\mathrm{CO}_{3}\right)_{2}(\mathrm{OH})_{2}$. J. Phys. Condens. Matter 2016, 28, 056002. [CrossRef] 